\title{
Aeroelastic and Aerothermoelastic Vehicle Behavior in Hypersonic Flow
}

\author{
Jack J. McNamara*, Peretz P. Friedmann† Kenneth G. Powell \\ Department of Aerospace Engineering, The University of Michigan, Ann Arbor, MI, 48109, USA \\ and \\ Robert E. Bartels \\ Aeroelasticity Branch, NASA Langley Research Center, Hampton, VA, 23681
}

\begin{abstract}
The aeroelastic and aerothermoelastic behavior of three-dimensional configurations in the hypersonic flow regime are studied. The aeroelastic behavior of a low aspect ratio wing, representative of a fin or control surface on a generic hypersonic vehicle, is examined using third order piston theory, Euler and Navier-Stokes aerodynamics. The sensitivity of the aeroelastic behavior generated using Euler and Navier-Stokes aerodynamics to parameters governing temporal accuracy is also examined. Also, a refined aerothermoelastic model, which incorporates the heat transfer between the fluid and structure using CFD generated aerodynamic heating, is used to examine the aerothermoelastic behavior of the low aspect ratio wing in the hypersonic regime. Finally, the hypersonic aeroelastic behavior of a generic hypersonic vehicle with a lifting-body type fuselage and canted fins is studied using piston theory and Euler aerodynamics for the range of $2.5 \leq \mathrm{M} \leq \mathbf{2 8}$, at altitudes ranging from $\mathbf{1 0 , 0 0 0}$ feet to $\mathbf{8 0 , 0 0 0}$ feet. This analysis includes a study on optimal mesh selection for use with Euler aerodynamics. In addition to the aeroelastic and aerothermoelastic results presented, three time domain flutter identification techniques are compared, namely the moving block approach, the least squares curve fitting method, and a system identification technique using an Auto-Regressive model of the aeroelastic system. In general, the three methods agree well. The system identification technique, however, provided quick damping and frequency estimations with minimal response record length, and therefore offers significant reductions in computational cost. In the present case, the computational cost was reduced by $75 \%$. The aeroelastic and aerothermoelastic results presented illustrate the applicability of the CFL3D code for the hypersonic flight regime.
\end{abstract}

\section{Nomenclature}

$a$

$a_{\infty}$

$A_{i}$

$\left\{\mathbf{A}_{\mathbf{p}}\right\}$

$a_{o}, a_{i}, b_{i}$

$b$

$c$
Nondimensional offset between the elastic axis and the midchord, positive for elastic axis behind midchord

Speed of sound

Constant in sinusoidal representation of transient modal amplitude

Estimated aeroelastic system matrix

Coefficients used for damping and frequency identification

Semi-chord

Reference length, chord length of double-wedge airfoil

\footnotetext{
*Ph.D. Candidate, Student Member AIAA.

${ }^{\dagger}$ François-Xavier Bagnoud Professor, Fellow AIAA.

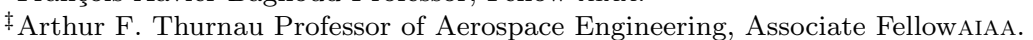

$\S$ Postdoctoral Fellow

"Senior Research Engineer
} 


\begin{tabular}{|c|c|}
\hline$C_{L}, C_{M}, C_{D}$ & Coefficients of lift, moment about the elastic axis, and drag \\
\hline$C_{p}$ & Coefficient of pressure \\
\hline$\left\{\mathbf{C}_{\mathbf{p}}\right\}$ & Estimated aeroelastic system matrix \\
\hline$c_{p w}$ & Specific heat of the wall \\
\hline$C F L_{\tau}$ & CFL3D input parameter regulating "pseudo time step size \\
\hline$E$ & Squared error between curve fit and actual data \\
\hline$f$ & Exponential function for curve fit \\
\hline$h$ & Airfoil vertical displacement at Elastic Axis \\
\hline$h_{i}$ & States in state-space representation of AR model \\
\hline$h_{h t}$ & Heat transfer coefficient \\
\hline$I_{\alpha}$ & Mass moment of inertia about the Elastic Axis \\
\hline$k$ & Discrete time \\
\hline$K_{\alpha}, K_{h}$ & Spring constants in pitch and plunge respectively; $K_{\alpha}=I_{\alpha} \omega_{\alpha}^{2}, K_{h}=m \omega_{h}^{2}$ \\
\hline$k_{\omega}$ & Reduced frequency \\
\hline$M$ & Free stream Mach number \\
\hline$m$ & Mass per unit span \\
\hline$M_{f}$ & Flutter Mach number \\
\hline $\mathbf{M}, \mathbf{K}$ & Generalized mass and stiffness matrices of the structure \\
\hline$N$ & Number of points in response signal \\
\hline $\mathbf{n}$ & Normal vector \\
\hline$N_{b}$ & Number of points in moving blocknumber \\
\hline$n_{m}$ & Number of modes used \\
\hline$p$ & Pressure \\
\hline$p_{\infty}$ & Free-stream pressure \\
\hline$q_{\infty}$ & Dynamic pressure \\
\hline Q & Generalized force vector for the structure \\
\hline$Q_{i}$ & Generalized force corresponding to mode $i$ \\
\hline$q_{i}$ & Modal amplitude of mode $i$ \\
\hline$\dot{q}_{\text {aero }}, \dot{q}_{\text {rad }}$ & Heat transfer rate due to aerodynamic heating, radiation, conduction, \\
\hline$q_{\text {cond }}, q_{\text {strd }}$ & and stored enegry respectively. \\
\hline$\underline{Q_{i}^{\prime}}$ & Finite Fourier transform of transient of mode $i$ \\
\hline$\overline{Q_{i}^{\prime}}$ & Amplitude of Fourier spectrum at one time-step (moving-block function) \\
\hline$r$ & Real part of eigenvalue \\
\hline$r_{\alpha}$ & Nondimensional radius of gyration \\
\hline$R e$ & Reynolds number \\
\hline$S$ & Surface area of the structure \\
\hline$s$ & Imaginary part of eigenvalue \\
\hline$S_{\alpha}$ & Static mass moment of wing section about elastic axis \\
\hline$T$ & Temperature \\
\hline$t$ & Time \\
\hline$\Delta t$ & Time step \\
\hline$T_{E}$ & Kinetic energy of the structure \\
\hline$T_{e}$ & Sample time \\
\hline$t_{h}$ & Airfoil half thickness \\
\hline$t_{k}$ & $k_{t h}$ point in discrete time \\
\hline$T_{R}$ & Radiation equilibrium wall temperature \\
\hline$T_{r}$ & Response signal length \\
\hline$T_{W}$ & Wall temperature \\
\hline$T_{A W}$ & Adiabatic wall temperature \\
\hline$T_{\text {Ref }}$ & Reference temperature \\
\hline$U_{E}$ & Potential energy of the structure \\
\hline$V$ & Free stream velocity \\
\hline$v_{n}$ & Normal velocity of airfoil surfaces \\
\hline$w$ & Displacement of the surface of the structure \\
\hline$x, y, z$ & Spatial Coordinates \\
\hline
\end{tabular}




\begin{tabular}{|c|c|}
\hline$x_{\alpha}$ & $\begin{array}{l}\text { Nondimensional offset between the elastic axis and the cross-sectional center of gravity, } \\
\text { positive for center of gravity behind elastic axis }\end{array}$ \\
\hline$\left\{X_{p}\right\}$ & State matrix \\
\hline$y^{+}$ & Law-of-the-wall coordinate \\
\hline$Z(x, y, t)$ & Position of structural surface \\
\hline$\alpha$ & Airfoil pitch displacement about the Elastic Axis \\
\hline$\alpha_{s}$ & Static angle of attack \\
\hline$\Delta_{w}$ & Skin thickness \\
\hline$\Delta_{k}$ & Nondimensional grid-spacing \\
\hline$\delta_{k-1}$ & Input for ARMA model of aeroelastic system \\
\hline$\epsilon$ & Emissivity \\
\hline$\gamma$ & Ratio of specific heats \\
\hline$\kappa$ & Thermal conductivity \\
\hline$\Lambda$ & Estimated matrix eigenvalue \\
\hline$\mu_{m}$ & Mass ratio \\
\hline$\omega_{\alpha}, \omega_{h}$ & Natural frequencies of uncoupled pitch and plunge motions \\
\hline$\omega_{i}$ & Frequency of mode $i$ \\
\hline$\omega_{n i}$ & Natural frequency of mode $i$ \\
\hline$\Phi$ & Modal matrix \\
\hline$\phi_{i}$ & Mode shape for mode $i$ \\
\hline$\phi_{a i}$ & Phase angle in radians of modal amplitude history in sinusoidal representation, for mode $i$ \\
\hline$\rho$ & Air density \\
\hline$\rho_{W}$ & Wall density \\
\hline$\sigma$ & Stefan-Boltzmann constant \\
\hline$\tau$ & Local inclination to the flow \\
\hline$\tau_{t}$ & Time \\
\hline$\zeta$ & Damping ratio \\
\hline$\dot{(}), \ddot{(})$ & First and second derivatives with respect to time \\
\hline
\end{tabular}

\section{Introduction}

TyPersonic aeroelasticity and aerothermoelasticity was an active area of research in the late 1950's and 1 during the 1960's as evident from Refs. 1-4. This research was instrumental in providing the basis for the aerothermoelastic design of the space shuttle. For a considerable time period there was only limited interest in this area until the advent of the National AeroSpace Plane (NASP).

In recent years, renewed activity in hypersonic flight research has been stimulated by the need for a low cost, reusable launch vehicle (RLV) and the recently completed Hyper-X program at NASA. Other activities are motivated by the design requirements for unmanned hypersonic vehicles that meet the needs of the US Air Force.

Vehicles in this category are based on a lifting body design. However, stringent minimum-weight requirements imply a degree of fuselage flexibility. Aerodynamic surfaces, needed for control, are also flexible. Furthermore, to meet the requirement of a flight profile that spans the Mach number range from 0 to 15 , the vehicle must withstand severe aerodynamic heating. These factors combine to produce unusual aeroelastic problems that have received only limited attention in the past. Furthermore, it is important to emphasize that testing of aeroelastically scaled wind tunnel models, a conventional practice in subsonic and supersonic flow, is not feasible in the hypersonic regime. Thus, the role of aeroelastic simulations is critical for this flight regime.

Previous studies in this area can be separated into several groups. The first group consists of studies focusing on panel flutter, which is a localized aeroelastic problem representing a small portion of the skin on the surface of the hypersonic vehicle. ${ }^{5-10}$ The second group of studies in this area was motivated by a previous hypersonic vehicle, namely the NASP. ${ }^{11-17}$ However, some of these studies dealt with the transonic regime, because it was perceived to be quite important. Spain et al. ${ }^{12}$ carried out a flutter analysis of all-movable NASP-like wings with slab and double-wedge airfoils, and found that using effective shapes for the airfoils obtained by adding the boundary layer displacement thickness to the airfoil thickness improved the overall agreement with experiments. Aerothermoelastic analyses of NASP-like vehicles found that aerodynamic 
heating altered the aeroelastic stability of the vehicle through the degradation of material properties and introduction of thermal stresses. ${ }^{15-17}$

The third group of studies is restricted to recent papers that deal with the newer hypersonic configurations such as the X-33 or the X-34. Reference 18 considered the X-34 launch vehicle in free flight at $M=8.0$. In Ref. 19, CFD-based flutter analysis was used for the aeroelastic analysis of the X-43 configuration, using system identification based order reduction of the aerodynamic degrees of freedom. Both the structure and the fluid were discretized using the finite element approach. It was shown that piston theory and ARMA Euler calculations predicted somewhat similar results. In Ref. 20 the aeroelastic stability of a generic hypersonic vehicle resembling the X-33 was considered using piston theory. In a sequel to this study ${ }^{21}$ the piston theory results for the aeroelastic behavior were also compared to aeroelastic stability boundaries obtained from computational aeroelasticity.

From the studies on previous hypersonic vehicles, ${ }^{11,19,22,23}$ one can identify operating envelopes for each vehicle. A graphical representation of these operating conditions is shown in Fig. 1. This figure is a convenient illustration of typical operating conditions of generic hypersonic vehicles.

The authors of this paper described an aeroelastic analysis capability for generic hypersonic vehicles in the Mach number range $0.5<M<15.0$, using computational aeroelasticity. ${ }^{24-26}$ The computational tool consisted of a combination of the CFL3D $\operatorname{code}^{27}$ and a finite element model of both a generic hypersonic vehicle, and three-dimensional wing, utilizing MSC.NASTRAN. This paper continues to explore fundamental aspects of hypersonic aeroelasticity and aerothermoelasticity using computational tools and it focuses on three-dimensional low aspect ratio wing and complete generic hypersonic vehicle configurations. The specific objectives of the paper are:

1. Compare the aeroelastic behavior predicted on a low aspect ratio wing using CFL3D, with both Euler and Navier-Stokes solutions for the unsteady aerodynamic loads. Examine the sensitivity of these computations to parameters relating to temporal accuracy.

2. Study different time domain damping and frequency identification techniques in order to assess the properties and effectiveness of these methods.

3. Obtain the stability boundaries for the low-aspect ratio wing using unsteady Euler and Navier-Stokes aerodynamics, and compare them with similar results based on third order piston theory.

4. Develope a refined aerothermoelastic model that incorporates the effect of heat transfer between the structure and the fluid due to intense aerodynamic heating.

5. Predict the aeroelastic behavior of a generic hypersonic vehicle using nonlinear piston theory and Euler aerodynamics over a broad range of Mach numbers and altitudes. Examine the sensitivity of the flutter prediction to grid resolution.

These objectives will significantly enhance our understanding of hypersonic aeroelasticity as applied to reusable launch vehicles.

\section{Method of Solution}

The computational aeroelastic solutions in the present study are obtained using the CFL3D code. ${ }^{27}$ The CFL3D code is used to generate both steady and unsteady air loads, and it also produces the aeroelastic transients and response solutions. The fluid/structure coupling in the code is accomplished using the free vibration modes of the vehicle.

\section{A. Euler/Navier-Stokes Solver in CFL3D}

The aeroelastic analysis in this study is carried out using the CFL3D code. The code uses an implicit, finite-volume algorithm based on upwind-biased spatial differencing to solve the time-dependent Euler and Reynolds-averaged Navier-Stokes equations. Multigrid and mesh-sequencing are available for convergence acceleration. The algorithm, which is based on a cell-centered scheme, uses upwind-differencing based on either flux-vector splitting or flux-difference splitting, and can sharply capture shock waves. For applications utilizing the thin-layer Navier-Stokes equations, different turbulence models are available. For time-accurate 
problems using a deforming mesh, an additional term accounting for the change in cell-volume is included in the time-discretization of the governing equations. ${ }^{28}$ Since CFL3D is an implicit code using approximate factorization, linearization and factorization errors are introduced at every time-step. Hence, intermediate calculations referred to as "subiterations" are used to reduce these errors. Increasing these subiterations improves the accuracy of the simulation, albeit at increased computational cost.

\section{B. Aeroelastic Option in CFL3D}

The aeroelastic approach underlying the CFL3D code is similar to that described in Refs. 29 and 30. The equations are derived by assuming that the general motion $w(x, y, z, t)$ of the structure is described by a finite modal series given by Eq. (1) below. The functions $\phi_{i}(x, y, z)$ represent the free vibration modes of the vehicle which are calculated using a finite element approach.

$$
w(x, y, z, t)=\sum_{i=1}^{n_{m}} q_{i}(t) \phi_{i}(x, y, z)
$$

The aeroelastic equations of motion are obtained from Lagrange's equations,

$$
\frac{d}{d t}\left(\frac{\partial T_{E}}{\partial \dot{q}_{i}}\right)-\frac{\partial T_{E}}{\partial q_{i}}+\frac{\partial U_{E}}{\partial q_{i}}=Q_{i}, \quad i=1,2, \ldots, n_{m}
$$

which yield

$$
\mathbf{M} \ddot{\mathbf{q}}+\mathbf{K q}=\mathbf{Q}(\mathbf{q}, \dot{\mathbf{q}}, \ddot{\mathbf{q}}), \quad \mathbf{q}^{T}=\left[q_{1} q_{2} \ldots q_{n_{m}}\right]
$$

where the elements of the generalized force vector are given by,

$$
Q_{i}=\frac{\rho V^{2}}{2} c^{2} \int_{S} \phi_{i} \frac{\Delta p(\mathbf{q}, \dot{\mathbf{q}}, \ddot{\mathbf{q}}) d S}{\rho V^{2} / 2 c^{2}}
$$

The aeroelastic equations are written in terms of a linear state-space equation (using a state vector of the form $\left[\begin{array}{llllll}\ldots & \dot{q}_{i-1} & q_{i} & \dot{q}_{i} & q_{i+1} & \ldots\end{array}\right]^{T}$ ) such that a modified state-transition-matrix integrator can be used to march

the coupled fluid-structural system forward in time. At the beginning of each time-step, the incremental structural deflections are calculated using the modal velocities and generalized aerodynamic forces. Using a deforming mesh, the mesh points are moved so that the inner mesh boundaries conform to the new deformed shape of the structure while the far-field boundaries are held stationary. The fluid equations, including the geometric conservation law terms, compute the flow field throughout the updated mesh. The generalized aerodynamic forces acting on the structure through the next time-step are then computed. Thus, a tight coupling of the flow and the structure is implemented through the generalized aerodynamic forces. Thus, a time-history of the modal displacements, modal velocities and generalized forces is obtained.

The aeroelastic capabilities of CFL3D, based on this modal response approach for obtaining the flutter boundary, have been partially validated for the transonic regime for the first AGARD standard aeroelastic configuration for dynamic response, Wing 445.6. The results of flutter calculations using Euler aerodynamics are given in Ref. 31 and those using Navier-Stokes aerodynamics are given in Ref. 32. For more recent validation using the AGARD 445.6 wing flutter onset envelop, the current version of CFL3D v6.0 was used in Ref. 33.

\section{Computational Methods for Fluid-Structure Coupling}

Prediction of the dynamic response of a flexible structure in a fluid requires the simultaneous solutions of the equations of motion of the structure and the fluid. In order to impose the kinematic boundary conditions on the fluid mesh at the new time step, the location and velocity of the fluid-structure boundary must first be known. This requires the solution of the entire system of equations for the structure, a task that cannot be carried out until the current surface pressure is known, which depends on the solution of the fluid domain and thus also on the unknown boundary conditions during the current time step. In addition, the discretized model of the structure uses a Lagrangian approach by following a point located on the structure over time, while the discretized model of the fluid uses an Eulerian approach by computing the flow quantities at a specific location in space over time. Therefore, accurate coupling of the two systems 
is a fairly complicated endeavor. A straightforward approach to the solution of the coupled fluid-structure system requires changing the fluid grid at each time-step, which is computationally very expensive. Therefore, several different approaches have emerged as alternatives to partial regridding in transient aeroelastic computations, among them being dynamic meshes ${ }^{34}$ the space-time formulation, ${ }^{35-37}$ the Arbitrary/Mixed Eulerian-Lagrangian formulation, ${ }^{38,39}$ the multiple-field formulation, ${ }^{40,41}$ the transpiration method, ${ }^{19,42}$ the subgrid/TFI method, ${ }^{43}$ and the modified spring analogy. ${ }^{28}$ A detailed description of these methods is provided in Ref. 44. However, since the CFL3D code used here implements fluid-structure coupling using the subgrid/TFI method and modified spring analogy, these methods are described in detail below.

The subgrid/TFI method is an algebraic mesh deformation method in which surface movement is transmitted with an exponential decay into the mesh interior. The motion of selected slave points, chosen across a grid at constant index intervals, is tied to the motion of the nearest surface (or master) point. The exponential decay function uses distance between the slave and nearest surface point so that motion of the surface is transmitted nearly undiminished to nearby slave points. On block faces, intervening mesh points are updated using transfinite interpolation (TFI). Once the intermediate mesh between slave points is updated on block faces, block interiors are updated using a volume TFI step. When smoothing of the deformed grid is necessary, several iterations of a modified spring analogy are employed. This scheme is a modification of the spring analogy ${ }^{34}$ using axial spring stiffness. Spring stiffness in the mesh interior is controlled by the spacing of the appropriate boundary grid points. Note that the axial stiffness approach results in smoothing of the mesh and also allows adaptation based on the flow solution. Furthermore, the problem of grid collapse around convex surfaces is handled by selectively increasing/decreasing stiffness based on surface curvature. Finally, the results generated using CFL3D in this study employ an improved scheme that ensures the generated control points at block boundaries are coincident and also checks block boundaries for separation during the mesh deformation at each time step. More details for this improved scheme will be available in Ref. 45.

\section{General Overview of the Solution Process}

The solution of the computational aeroelasticity problem used in the present study is shown in Fig. 2. First, the vehicle geometry is created using CAD software, and from this geometry a mesh generator is used to create a structured mesh for the flow domain around the body. In parallel, an unstructured mesh is generated that represents the surface of the finite element model of the vehicle. This structural model is further refined with internal stiffeners and mass elements to represent a complete vehicle, and is used to obtain the free vibration modes of the vehicle using MSC NASTRAN. Subsequently, the fluid mesh is used to compute the flow around the rigid vehicle using a CFD solver, which consists of the CFL3D code developed by NASA Langley Research Center. In order to generate the aeroelastic input for CFL3D, the modal deformation at each surface gridpoint in the fluid mesh is obtained by using cubic interpolation (in MATLAB) from the finite element structural model, for each structural mode. Using the flow solution as an initial condition, and the interpolated modal data as additional input, an aeroelastic equilibrium state is obtained for the flexible vehicle. For a geometry with vertical symmetry at zero angle of attack, such as a double-wedge airfoil, the equilibrium state is the same as the undeflected state. Next, the structure is perturbed in one or more of its modes by an initial modal velocity condition, and the transient response of the structure is obtained. To determine the flutter conditions at a given altitude, aeroelastic transients are computed at several Mach numbers and the corresponding dynamic pressures. The frequency and damping characteristics of the transient response for a given flight condition and vehicle configuration can be determined using some time domain damping and frequency identification method. This approach applied at the same altitude and vehicle configuration for a range of Mach numbers results in a series of damping values for the system. The flutter Mach number can be estimated from this series by interpolating the damping data points to identify the value of the Mach number at which the damping is zero.

\section{E. Time Domain Damping and Frequency Identification}

As mentioned earlier, the aeroelastic behavior of a configuration is obtained from CFL3D as a set of aeroelastic transient responses; from these the damping and frequency characteristics of the system are determined in the time domain. Two commonly used time domain identification methods are the moving block approach $(\mathrm{MBA})^{46,47}$ and the least squares curve fitting method (LSCFM). ${ }^{48} \mathrm{~A}$ more recent method performs system identification of an Auto-Regressive Moving Average (ARMA) model of the system. ${ }^{49-51}$ 
The MBA, originally developed in the early 1970's, uses the finite Fourier transform of a discretely sampled transient signal to identify modal damping and frequency. Consider a transient signal given by:

$$
q_{i}(t)=A_{i} e^{-\zeta_{i} \omega_{n i} t} \sin \left(\omega_{i} t+\phi_{a i}\right)
$$

where

$$
\omega_{i}^{2}=\omega_{n i}^{2}\left(1-\zeta_{i}^{2}\right)
$$

Then, the finite Fourier transform of this signal is:

$$
Q_{i}^{\prime}\left(\omega_{i}, \tau_{t}\right)=\int_{\tau_{t}}^{\tau_{t}+T_{r}} A_{i} e^{-\zeta_{i} \omega_{n i} t} \sin \left(\omega_{i} t+\phi_{a i}\right) e^{-i \omega_{i} t} d t
$$

where $Q_{i}^{\prime}\left(\omega_{i}, \tau_{t}\right)$ is a function of $\tau_{t}$ at the analysis frequency, $\omega_{i}$. The moving block function is defined as the amplitude of Eq. (7). When the damping is small, i.e. $\zeta_{i} \ll 1$, the natural logarithm of the moving block function is given by:

$$
\ln \overline{Q_{i}^{\prime}}\left(\omega_{i}, \tau_{t}\right)=-\zeta_{i} \omega_{i} \tau_{t}+\frac{1}{2} \zeta_{i} \sin \left\{2\left(\omega_{i} \tau_{t}+\phi_{a i}\right)\right\}+\text { constant }
$$

Note that Eq. (8) has a linear component with a slope of $-\zeta_{i} \omega_{i}$, and an oscillatory component at twice the analysis frequency, $\omega_{i}$.

The method is applied by using a Fast Fourier Transform (FFT) algorithm on the entire transient response to calculate the frequency spectrum of the signal, from which a frequency of interest, or analysis frequency, is selected. Next, Eq. (8) is calculated on a block of the data, where $N_{b}<N$, at $\tau_{t}=0$. This is repeated $N-N_{b}$ times, after shifting the block one sample point at a time, i.e. $\tau_{t}=n \Delta t$ for $n=0,1,2, \ldots, N-N_{b}$. The slope of Eq. (8) is calculated from the resulting curve using a least-squares curve fit, and the damping is calculated by dividing this slope by the analysis frequency.

A critical step with this method is the selection of an analysis frequency from the frequency spectrum of a given response. Since the aeroelastic response of the system is produced as a set of transient modal amplitudes, there are two options. One option is to calculate the frequency spectrum of the response from the transient displacement, Eq. (1), of a point on the structure. The analysis frequencies of the displacement will correspond to local maxima in the frequency spectrum. Image processing, therefore, is required, either by the user, or by an image processing subroutine, to locate the frequencies. The second option for locating the frequencies is to calculate the frequency spectrum of each transient modal amplitude individually. However, since the modal amplitudes are coupled through the generalized forces, the frequency spectrum of each modal amplitude will contain multiple frequencies. The analysis frequency, therefore, should be selected as the global maximum from the frequency spectrum of each modal amplitude. Note, however, that as flutter is approached, the frequencies of some modal amplitudes can become dominant throughout the system. When this occurs, selecting the global maximum from the frequency spectrum results in sudden shifts of the damping and frequency of some of the transients, since the MBA is tracking a different component. While information of some of the modes is lost when this occurs, this option provides the analyst insight as to how the modes interact as the flutter Mach number is approached.

There are a two main issues with the MBA for damping and frequency identification. In cases where a particular mode damps out rapidly, if the response record is too long, the moving block function will no longer form a straight line, and therefore the damping, which is calculated from a linear least squares fit of the MBA output data, will be incorrect. Since the amount of damping in each mode is not known before a response is processed, this issue introduces uncertainty in appropriate record lengths for accurate damping estimates. A second issue is the inability to identify peaks in the frequency spectrum when there are closely spaced modal frequencies.

The LSCFM, ${ }^{48}$ also developed in the 1970's, performs a least squares curve fit of a transient response to exponential functions in order to identify modal damping and frequency.

Consider the following complex exponential function:

$$
f(t)=a_{0}+\sum_{i=1}^{n_{m}} e^{-\zeta_{i} \omega_{n i} t}\left\{a_{i} \cos \left(\omega_{i} t\right)+b_{i} \sin \left(\omega_{i} t\right)\right\}
$$


where $n_{m}$ represents the number of modes in the signal. The modal damping and frequency of a transient response can be calculated by minimizing the squared-error difference between the output fit $f\left(t_{k}\right)$ and the input transient response $w\left(t_{k}\right)$, where $t_{k}$ is the $k^{t h}$ discrete time sample of the response. This error is given by:

$$
E=\sum_{k=1}^{N}\left\{f\left(t_{k}\right)-w\left(t_{k}\right)\right\}^{2}
$$

Note that this is a nonlinear least squares problem. One method of solution is to provide initial "guess" values for $\zeta_{i}$ and $\omega_{i}$, and then solve the linear least squares problem for $a_{0}, a_{i}$, and $b_{i}$. The curve fit is then calculated by searching for the $\zeta_{i}$ and $\omega_{i}$ which minimize Eq. (10). At each search step the linear coefficients $\left(a_{0}, a_{i}\right.$, and $\left.b_{i}\right)$ are updated, and the error is recomputed, using the current $\zeta_{i}$ and $\omega_{i}$. The search for $\zeta_{i}$ and $\omega_{i}$ in this study is performed using the subroutine FMINSEARCH in MATLABC.

The weakness of this method is the computational time required to find the $a_{0}, a_{i}$, and $b_{i}$, which minimize Eq. (10), particularly when more than two modes are present in the aeroelastic system.

The ARMA system identification method for damping and frequency identification ${ }^{49-51}$ is based on a single-input single-output deterministic ARMA model of the aeroelastic system, with $2 n_{m}$ Auto-Regressive (AR) and one Moving Average (MA) coefficients. The model has the following form:

$$
q_{k}+\sum_{i=1}^{2 n_{m}} a_{i} q_{k-i}=b_{1} \delta_{k-1}
$$

where $2 n_{m}$ AR coefficients are used to determine the aeroelastic system damping and frequencies, and one MA coefficient is sufficient for identifying an aeroelastic static offset. To identify the damping and frequency, let the transient aeroelastic response be represented by the following AR model:

$$
q_{k}+\sum_{i=1}^{2 n_{m}} a_{i} q_{k-i}=0
$$

This can be written in state-space form as:

$$
\begin{aligned}
\left\{X_{p}\right\}_{k+1} & =\left\{\mathbf{A}_{\mathbf{p}}\right\}\left\{\mathbf{X}_{\mathbf{p}}\right\}_{\mathbf{k}} \\
q_{k} & =\left\{\mathbf{C}_{\mathbf{p}}\right\}\left\{\mathbf{X}_{\mathbf{p}}\right\}_{\mathbf{k}}
\end{aligned}
$$

where

$$
\left\{\mathbf{A}_{\mathbf{p}}\right\}=\left[\begin{array}{ccccc}
-a_{1} & 1 & 0 & \cdots & 0 \\
-a_{2} & 0 & 1 & \cdots & 0 \\
\vdots & \vdots & \vdots & \ddots & \vdots \\
-a_{2 n_{m}-1} & 0 & 0 & \cdots & 1 \\
-a_{2 n_{m}} & 0 & 0 & \cdots & 0
\end{array}\right]
$$

and

$$
\left\{\mathbf{C}_{\mathbf{p}}\right\}=\left[\begin{array}{lllll}
1 & 0 & 0 & \cdots & 0
\end{array}\right]
$$

The state vector, $\left\{X_{p}\right\}_{k}$, is defined as

$$
\left\{X_{p}\right\}_{k} \equiv\left\{\begin{array}{c}
q_{k} \\
h_{1}(k) \\
\vdots \\
h_{2 n_{m}-1}(k)
\end{array}\right\}
$$


with

$$
\begin{aligned}
q_{k} & =-a_{1} q_{k-1}+h_{1}(k-1) \\
h_{1}(k) & =-a_{2} q_{k-1}+h_{2}(k-1) \\
& \vdots \\
h_{2 n_{m}-2}(k) & =-a_{2 n_{m}-1} q_{k-1}+h_{2 n_{m}-1}(k-1) \\
h_{2 n_{m}-1}(k) & =-a_{2 n_{m}} q_{k-1}
\end{aligned}
$$

Note that the state-space description of the AR model in Eq. (13) is in observer form and is completely observable. The aeroelastic system damping and frequencies are determined from the eigenvalues of the estimated matrix, $\left\{\mathbf{A}_{\mathbf{p}}\right\}$, given in Eq. (14). These can be written as:

$$
\begin{aligned}
\Lambda_{j} & =r_{j}+i s_{j} \\
\Lambda_{j+n_{m}} & =r_{j}-i s_{j}
\end{aligned}
$$

where $j=1,2, \cdots, n_{m}$. Note that the AR model is in the discrete time domain, therefore, the aeroelastic modal damping and frequencies in the continuous time domain are given by:

$$
\begin{aligned}
\zeta_{j} & =\frac{1}{2 T_{e}} \log _{e}\left(r_{j}^{2}+s_{j}^{2}\right) \\
\omega_{j} & =\frac{1}{T_{e}} \tan ^{-1} \frac{s_{j}}{r_{j}}
\end{aligned}
$$

In this study, the AR coefficients are determined from the aeroelastic responses using the subroutine AR from the system identification toolbox in MATLAB(C). Note that the sample times, used to calculate the AR coefficients from the response data, were set to:

$$
T_{e}=\frac{\pi}{2\left(\omega_{n}\right)_{\max }}
$$

where $\left(\omega_{n}\right)_{\max }$ is the maximum system natural frequency in radians/second.

\section{F. Computational Model for the Three-Dimensional Low Aspect Ratio Wing}

Few studies ${ }^{52,53}$ have been carried out that validate CFL3D for the hypersonic regime. In part, this is because CFL3D was not originally designed for modeling hypersonic flow, and does not contain the necessary ingredients for computing real-gas effects. However, depending on the cases considered, and the most relevant flow variables, sufficiently accurate solutions can be obtained while ignoring real gas effects. References 52 and 53 compared the pressure distributions and static and dynamic stability derivatives of cones and ogivecylinder bodies obtained using CFL3D with results obtained using a unified hypersonic/supersonic panel method in the range $3<M<6$. However, no attempt was made in these studies to compare aeroelastic stability boundaries obtained with CFL3D.

A systematic validation of the CFL3D code for flutter analysis in the hypersonic regime has never been undertaken. To validate aeroelastic stability boundaries computed with CFL3D, it is important to identify and use simple configurations for which aeroelastic stability boundaries can be computed using alternative approaches. Specifically, generating results for simple three-dimensional configurations using Euler and Navier-Stokes unsteady aerodynamic loads, and comparing them with results obtained using an independently developed aeroelastic code based on piston theory, represents a validation of CFL3D in the hypersonic regime.

The computational model for the low aspect ratio wing, shown in Figs. 3 and 4, was developed from a study $^{26}$ of grid configurations, where the appropriate computational domain and mesh resolution required for the hypersonic aeroelastic analysis of the low aspect ratio wing were determined. In Ref. 26, cell distribution and grid resolution were varied to construct a grid for the hypersonic flow regime that accurately and efficiently captured the flow. Each span section plane of the computational domain extends one-half chord-lengths downstream. The boundary of the grid surrounding the wing, from the leading edge to midchord, extends to a distance $10 \%$ beyond the shock that forms at $M=5.0$. The computational domain in the spanwise direction also extends beyond the tip of the wing by $35 \%$ of the semi-span length. Furthermore, the grid is tapered, in all three dimensions, so as to be compatible with the geometric taper of the wing. With $0.63 \times 10^{6}$ cells, this grid is a $57 \times 353 \times 33 \mathrm{C}-\mathrm{H}$-grid with 353 points around the wing and its wake $(289$ 
points on the wing surface), 57 points extending spanwise from the root (49 points on the wing on the wing surface), and 33 points extending radially outward from the surface.

\section{G. Computational Model for the Generic Hypersonic Vehicle}

In order to carry out aeroelastic simulations of the generic vehicle, shown in Fig. 5, using Euler aerodynamics, various meshes were constructed for the computational domain shown in Fig. 6. Developing a suitable mesh for use with CFL3D requires balancing between various conflicting constraints. One of the main factors governing the usefulness of the mesh is the overall size of the mesh, which directly affects runtime. Having fixed the overall mesh size, obtaining accurate results requires having optimum placements for the internal gridpoints. This is done by having the maximum possible number of gridpoints lie within the shocks present around the vehicle. The midplane of the selected mesh is shown in Fig. 7, while Fig. 8 shows the gridpoints around the typical cross-section of the generic vehicle. On the surface of the generic vehicle, the gridpoints are again finely spaced close to the surface of the vehicle.

By varying the nondimensional grid-spacing, representing the distance of the closest grid-point in the normal direction, different pressure distributions on the surface of the vehicle's canted fin, at $75 \%$ of span, were obtained in order to assess convergence. The grid size of $3 \times 10^{6}$ gridpoints, the gridpoint placement on the surface and all other grid parameters were kept constant for these simulations. As seen in Table 1 , the error in the moment acting on the typical section of the canted fin was reduced with decreasing $\Delta_{k}$. Reference 26 indicates that the maximum error of $0.28 \%$ is small enough to expect converged results. However, aeroelastic simulations could not be carried out for $\Delta_{k}$ less than $5 \times 10^{-7}$, as this produces mesh entanglement. When this occurs, cell boundaries penetrate through adjacent cell volumes. In CFL3D, this generates negative cell volumes in the region surrounding the entanglement. Reducing the time-step enables the use of slightly smaller grid spacing, but with a significant increase in computational time. Hence, the time-step size was selected to be $\Delta t=0.35 \times 10^{-3}$ sec, which allows accurate calculation of the aerodynamic forces, provides at least 50 time-steps per cycle of the highest frequency mode, and avoids mesh entanglement for this value of $\Delta_{k}$. The above mesh was referred to as the "fine" mesh, and for comparison, an additional mesh having $4 \times 10^{5}$ gridpoints was also created. This mesh is referred to as the "coarse" mesh, and has a nondimensional grid-spacing at the surface in the coarse grid of $\Delta_{k}=6.5 \times 10^{-6}$.

The coarse mesh is a $65 \times 177 \times 33 \mathrm{C}$-H-grid with 177 points around the vehicle and its wake, 65 points spanning the grid from side to side, and 33 points extending radially outward from the vehicle surface. Of these, 145 points were wrapped around the fuselage in the streamwise direction, while 33 points were distributed on the vehicle fuselage from side to side. The fin surfaces were discretized using 9 grid points in the span-wise direction and 17 grid points from leading edge to trailing edge. The refined mesh is a $129 \times 355 \times 65 \mathrm{C}$-H-grid with 355 points around the vehicle and its wake, 129 points spanning the grid from side to side, and 129 points extending radially outward from the vehicle surface. Of these, 289 points were wrapped around the fuselage in the streamwise direction, while 65 points were distributed on the vehicle fuselage from side to side. The fin surfaces were discretized using 17 grid points in the span-wise direction and 33 grid points from leading edge to trailing edge. In both cases, the computational domain was extended 0.5 vehicle-length upstream and 1.5 chord lengths downstream. On either side, the domain extended 0.5 vehicle-lengths spanwise. The distance to the upper and lower boundaries was selected to be slightly larger than the shocks forming at $M=5.0$, with the shocks from the nose of the vehicle flowing out of the domain boundaries without reflection.

\section{H. Piston Theory Aerodynamics}

Piston theory is a simple inviscid unsteady aerodynamic theory, that has been used extensively in supersonic and hypersonic aeroelasticity. It provides a point-function relationship between the local pressure on the surface of the vehicle and the component of fluid velocity normal to the moving surface. ${ }^{54,55}$ The derivation utilizes the isentropic "simple wave" expression for the pressure on the surface of a moving piston,

$$
\frac{p(x, t)}{p_{\infty}}=\left(1+\frac{\gamma-1}{2} \frac{v_{n}}{a_{\infty}}\right)^{\frac{2 \gamma}{(\gamma-1)}}
$$

where

$$
v_{n}=\frac{\partial Z(x, y, t)}{\partial t}+V \frac{\partial Z(x, y, t)}{\partial x}
$$

10 of 48 
The expression for piston theory is based on a binomial expansion of Eq. (21), where the order of the expansion is determined by the ratio of $\frac{v_{n}}{a_{\infty}}$. Reference 55 suggested a third-order expansion, since it produced the smallest error of the various orders of expansion used when compared to the limiting values of pressure, namely the "simple wave" and "shock expansion" solutions. The third-order expansion of Eq. (21) yields third order piston theory:

$$
p(x, t)-p_{\infty}=p_{\infty}\left[\gamma \frac{v_{n}}{a_{\infty}}+\frac{\gamma(\gamma+1)}{4}\left(\frac{v_{n}}{a_{\infty}}\right)^{2}+\frac{\gamma(\gamma+1)}{12}\left(\frac{v_{n}}{a_{\infty}}\right)^{3}\right]
$$

Combining Eqns. 1, 4, 22, and 23 yields the generalized forces obtained from piston theory.

\section{Refined Aerothermoelastic Model}

A realistic aeroelastic model for the hypersonic regime must include aerodynamic heating effects. Aerodynamic heating significantly alters the flow properties, ${ }^{56}$ degrades the material properties and also introduces thermal stresses. ${ }^{57-59}$ Aerodynamic heating of the flow surrounding the vehicle leads to significantly different thermodynamic and transport properties, high heat-transfer rates, variable $\gamma$, possible ionization, and nonadiabatic effects from radiation. ${ }^{56,57}$ Thermal stresses can arise from rapidly changing conditions of heat input where time lags are involved, or from equilibrium conditions of non-uniform temperature distribution. ${ }^{58,59}$ Commonly, the heated structure has lowered stiffness due to material degradation and thermal stresses, which manifest themselves as a reduction in frequencies. ${ }^{58-60}$

Previously, the authors of this study performed an exploratory aerothermoelastic analysis on a low aspect ratio cantilevered wing. ${ }^{26}$ As a first approximation to the aerothermoelastic system, the heating distribution of the system was assumed to be a parabolic function of the chord. Furthermore, steady-state conditions were assumed, resulting in a unique temperature distribution for each Mach number. An exact treatment of aerothermoelasticity requires the coupling of the unsteady heat transfer problem with the aeroelastic problem based on a Navier-Stokes solution of the unsteady airloads, which results in time dependent temperature distributions. This implies time dependent free vibration characteristics of a structure, at a given Mach number, as it is heated.

The heat transfer between the fluid and the structure, schematically depicted in Fig. 9, is determined from an energy balance of the heat fluxes at the wall of the structure: ${ }^{61,62}$

$$
\dot{q}_{\text {aero }}=\dot{q}_{\text {rad }}+\dot{q}_{\text {cond }}+\dot{q}_{\text {strd }}
$$

where

$$
\begin{gathered}
\dot{q}_{a e r o}=h_{h t}\left(T_{A W}-T_{W}\right) \\
\dot{q}_{r a d}=\sigma \epsilon T_{W}^{4} \\
\dot{q}_{c o n d}=\left(\kappa \frac{\partial T}{\partial \mathbf{n}}\right)_{W} \\
\dot{q}_{s t r d}=\rho_{W} c_{p w} \Delta_{w} \frac{d T_{W}}{d t}
\end{gathered}
$$

The heat transfer represents a balance at the wall between the convective heating by the fluid $\left(\dot{q}_{a e r o}\right)$ and heat loss due to conduction into the structure $\left(\dot{q}_{\text {cond }}\right)$, radiation out to space $\left(\dot{q}_{\text {rad }}\right)$, and energy stored in the wall $\left(\dot{q}_{s t r d}\right)$. The heat transfer problem is driven by Eq. 25 , or more specifically the adiabatic wall temperature, $T_{A W}$, which is a function of the surface geometry and free stream conditions. Note that at steady-state,

$$
\dot{q}_{a e r o}=\dot{q}_{\text {rad }}
$$

Therefore, using Eqs. 25 and 26,

$$
\sigma \epsilon T_{R}^{4}-h_{h t} T_{R}-h_{h t} T_{A W}=0
$$

where, given $h_{h t}, T_{A W}, \sigma$, and $\epsilon$, the radiation equilibrium temperature at the wall, $T_{R}$, can be determined by solving a 4 th order algebraic equation. 
The solution procedure for the refined aerothermoelastic system is depicted in Fig. 10. Comparing Figs. 2 and 10, the modification introduced by the aerothermoelastic solution is due to the fact that the aerodynamic heating information, namely $T_{A W}$ and $h_{h t}$ from Eq. 25, obtained on rigid body CFD computations, is introduced into the finite element analysis of the system. Specifically, $T_{A W}$ is calculated by specifying an adiabatic wall as a boundary condition for the CFD solution to the Navier-Stokes equations, and $h_{h t}$ is calculated from a separate Navier-Stokes solution where the wall temperature boundary condition is set to free stream temperature. Setting a free stream, or "cold", wall boundary condition to calculate $h_{h t}$ provides a conservative estimate of the aerodynamic heating. The transient temperature distribution in the structure is determined from a heat transfer analysis using finite element analysis, and the free vibration frequency and mode shapes of the transiently heated structure are then calculated at each desired point in time. This process is depicted in Fig. 11.

Note that this is not an exact treatment of the aerothermoelastic system, since the temperature distribution is not computed at each time step of the aeroelastic calculation procedure. It is, however, a reasonable approximation since the heat loads vary slowly with time compared to the generalized forces and motion of the system. Furthermore, it is computationally prohibitive to generate an aeroelastic computation continuously for the time scales involved to heat the structure.

The aerothermodynamic quantities in this study were determined using a $2.1 \times 10^{6}$ cell grid using CFL3D to solve the Navier-Stokes equations. The heat transfer analysis was performed using the "Transient Analysis Solution" (Sol 159) ${ }^{63}$ in MSC.NASTRAN with the "HEAT" option selected. The effect of thermal radiation was included. The heated modes and frequencies of the wing were determined using the "Nonlinear Statics Solution" in MSC.NASTRAN (Sol 106) ${ }^{63}$ with the "NORMAL MODES" option selected. The analysis includes both the effect of material property degradations and thermal stresses.

\section{Results and Discussion}

Comparison of aeroelastic stability results based on Euler, Navier-Stokes and piston theory in Refs. 24-26 provided useful guidelines regarding the importance of viscosity and aerodynamic heating, and the effectiveness of piston theory in approximating the aeroelastic behavior of simple two- and three-dimensional lifting surfaces. A similar approach is pursued here for three-dimensional low aspect ratio wings. First, however, the effectiveness of three time domain damping and frequency identification techniques is studied using aeroelastic data generated from a simple typical section analysis using unsteady Navier-Stokes aerodynamics. ${ }^{25}$ Next, the low aspect ratio wing is used to compare the aeroelastic behavior generated using third order piston theory, Euler and Navier-Stokes aerodynamics. Furthermore, the sensitivity of the aeroelastic behavior to several parameters related to time stepping is studied using Euler aerodynamics. Next, the aerothermoelastic behavior of a low aspect ratio wing is studied using the refined aerothermoelastic model. Finally, the aeroelastic behavior of a generic hypersonic vehicle is examined using third order piston theory and Euler aerodynamics at various altitudes. This configuration is also used to study the sensitivity of the aeroelastic behavior to grid resolution using Euler aerodynamics.

\section{A. Comparison of Time Domain Frequency and Damping Identification Techniques}

The three identification methods (MBA, LSCFM, ARMA) have been previously compared, in Ref. 50, using several damped free oscillation examples, as well as subsonic and transonic aeroelastic data. The ARMA method was found to be superior for the cases considered. ${ }^{50}$ Specifically, the ARMA model calculated the damping and frequency of modes with only $0.01 \%$ separation in frequencies. Furthermore, the ARMA model required substantially smaller time records than either the MBA or LSCFM to accurately extract system damping and frequencies. For computational aeroelasticity, this is an important consideration when choosing a time domain flutter identification technique since the cost of a computational aeroelastic simulation is largely determined by the size of the response record required to calculate system damping.

To compare these three methods, each was used to calculate the frequency and damping for a simple typical section aeroelastic system, shown in Fig. 12. The parameters describing this configuration are listed in Table 2. The aeroelastic responses were generated by CFL3D using unsteady Navier-Stokes aerodynamics at an altitude of 40,000 ft. The computational domain used for the CFD computations of the typical section was created from the $75 \%$ span section of the low aspect ratio wing computational domain. The aeroelastic transients were calculated for 4000 time steps, corresponding to 1 second of response. A comparison of each 
method is given in Fig. 13. For the results labeled ARMA, MBA, and LSCFM, the damping and frequency of the system were calculated from the pitch response of the system, where:

$$
\left\{\begin{array}{l}
h(t) \\
\alpha(t)
\end{array}\right\}=[\mathbf{\Phi}]\left\{\begin{array}{l}
q_{1}(t) \\
q_{2}(t)
\end{array}\right\}
$$

The results labeled MBA2 were generated using the individual aeroelastic transients, i.e. $\left\{q_{1}(t), q_{2}(t)\right\}$. The MBA results, therefore, were generated using the MATLAB@ subroutine IMREGIAONALMAX to locate the analysis frequencies from the frequency spectrum of the pitch response. The MBA2 results were generated using the global maximum from the frequency spectrum of each individual transient as the analysis frequencies.

It is evident that all three methods calculate similar damping and frequency estimates for the typical section responses. In particular, each method predicts the same flutter Mach number, $M_{f}=11.9$, when the damping becomes zero. Furthermore, the ARMA, LSCFM, and MBA/MBA2 methods predict the same aeroelastic behavior, over the range of Mach numbers considered, for the second mode. The behavior of the first mode, i.e. lower frequency mode, is calculated equivalently using both ARMA and LSCFM. The MBA and MBA2 methods show the behavior of the first mode as approaching the second mode as the flutter Mach number is approached. Specifically, for the MBA2 method, the aeroelastic behavior of the first mode is similar to the second mode from $M=11.0$ up to flutter. Frequency spectrum plots of the transients, shown in Figs. 14 and 15, reveal that the dominant frequency (i.e. global maximum of FFT) of the first modal transient shifts from the first modal frequency to the second modal frequency between $M=10.0$ and $M=11.0$. This indicates that the second modal frequency dominates both the first and second modal transients as flutter is approached. For the MBA method, the damping of the first mode approaches the damping of the second mode from $M=11.0$ to $M=11.75$. Beyond $M=11.75$, no analysis frequency is available for the first mode. Figures 16 and 17 illustrate that at $M=11.0$, two local peaks are present in the frequency spectrum of the pitch response. However, at $M=11.75$, the modal frequencies have coalesced to a point where the local peak corresponding to the first modal frequency is no longer identifiable by the image processing routine, IMREGIONALMAX. This illustrates the failure of this method to identify closely spaced analysis frequencies.

The performance of each method as the time record of the response is reduced is illustrated in Figs. 18 - 21. It is evident from these results that, for this aeroelastic system, both the ARMA method and the LSCFM are capable of computing the aeroelastic behavior accurately when the time record is reduced substantially. In this case, the aeroelastic behavior is calculated from these two methods with only 250 time steps, corresponding to 0.06 seconds. However, the performance of the MBA and MBA2 methods diminishes as the time record is shortened. These methods require at least 0.25 seconds of response data to provide reasonable estimations for the damping and frequency. Furthermore, the estimated behavior, using ARMA and LSCFM, is invariant when the response record is shortened, while there is some variation in the MBA/MBA2 results as the record length is changed. In both the MBA/MBA2 results, the flutter Mach number is slightly reduced, and the behavior before flutter varies, as the record length is shortened.

In the cases considered here, the ARMA method is superior to both the MBA and LSCFM. It produces damping and frequency estimates from time records that are $75 \%$ shorter than those required for the MBA. Furthermore, it is superior to the LSCFM in terms of computational efficiency. The ARMA method required $\sim 3$ seconds on a $3.06 \mathrm{GHz}$ Xeon processor, while, depending on the size of the time record used, the LSCFM required up to 5 minutes of CPU time. Furthermore, the time required for the ARMA method to generate damping and frequency estimations is relatively independent on the number of modes tracked in the system, while the time required for the LSCFM increases with the number of modes tracked.

\section{B. Aeroelastic Behavior of a Three-Dimensional Low Aspect Ratio Wing}

The parameters describing the physical properties of the three-dimensional low aspect ratio wing are provided in Table 3 and Fig. 22. The natural frequencies and modes, shown in Fig. 23, were determined by comparing them with the bending and torsional frequencies and total mass of a wing that resembles the Lockheed F-104 wing. For the Euler and Navier-Stokes computations, the time step size was set to accommodate 50 steps per cycle of the highest frequency mode, which corresponds to $\Delta t=0.25 \times 10^{-3}$ seconds. This resulted in a smaller time step than that required for modeling the unsteady aerodynamic loads. ${ }^{25}$ 
The sensitivity of the aeroelastic behavior of the wing to the number of modes is illustrated in Table 4 . It is evident that increasing the number of modes from 5 to 8 has little effect on the aeroelastic behavior when third order piston theory aerodynamics is used. However, it is also clear that at least five modes are needed to capture the aeroelastic behavior. The aeroelastic behavior of the low aspect ratio wing using third order piston theory with 5 modes, at 40,000 ft, is shown in Fig. 24, where $M_{f}=13.4$. Using the frequency of the flutter mode, this corresponds to $k_{\omega}=0.14$, while for Mode $5, k_{\omega}=0.51$. For this case, as the Mach number is increased, there is a coalescence in frequency for the first bending (Mode 1) and first torsion (Mode 2) modes, while the damping of the first mode approaches zero. Also, as the damping of Mode 1 decreases, the damping of Mode 2 increases. The damping and frequency of the Modes $3-5$, are relatively constant with increasing Mach number. Note that the ARMA system identification method was used to calculate the damping and frequency of the system.

In order to solve the unsteady Euler and Navier-Stokes equations, several temporal parameters must be set in CFL3D so as to conduct efficient time-accurate calculations. Specifically, CFL3D employs a "pseudo" time step ${ }^{27}$ in order to reduce linearization and factorization errors in the time-accurate computations. The "pseudo" time step is implemented by specifying a number of sub-iterations, as well as the "pseudo" time step size, which is governed by $C F L_{\tau}$. In general, decreasing the global time step reduces the number of sub-iterations required to achieve an accurate result, while increasing the grid refinement increases the number of sub-iterations required. ${ }^{27}$ The effect of sub-iterations and $C F L_{\tau}$ on the hypersonic aeroelastic behavior of the low aspect ratio wing is illustrated in Fig. 25 using a $0.63 \times 10^{6}$ cell grid. These results were generated at 40,000 ft, for $M=12.0$, using Euler aerodynamics. This Mach number and altitude were chosen since it was expected, based on the piston theory results, to result in aeroelastic behavior that was relatively close to, but not above, the flutter boundary. It is evident from Fig. 25 that the number of sub-iterations used has an effect on the aeroelastic behavior of the wing. While the frequencies of all the modes remain constant, the damping varies with increasing sub-iterations. In particular, the first and second modes are substantially affected by the number of sub-iterations. The most significant impact is the relative magnitude of the damping, where the first and second modes cross each other depending on the number of sub-iterations used. For $C F L_{\tau}=1.0$, the damping of the second mode is greater than the damping of the first mode, based on 25 - 45 sub-iterations. Furthermore, the damping of all the modes remains almost constant in this range of sub-iterations. Also, when $C F L_{\tau}=5.0$, which represents a larger step in "pseudo-time" per sub-iteration, the same behavior is observed, but for a smaller number of sub-iterations. In the $C F L_{\tau}=5.0$ case, when the number of sub-iterations varies between 20 - 35, the behavior is relatively unchanged. Based on these results, $C F L_{\tau}=5.0$ with 20 sub-iterations is selected since it provides the most efficient and accurate results when Euler aerodynamics are used. As illustrated in Fig. 26, the flutter Mach number of the low aspect ratio wing at 40,000 ft is $M_{f}=13.7\left(0.15<k_{\omega}<0.49\right)$ when Euler aerodynamics are used. Note that this is only $2 \%$ higher than the flutter Mach number predicted using third order piston theory. Also, similar to the piston theory results, there is a coalescence in frequency between the first bending (Mode 1) and first torsion (Mode 2) modes, while the damping of the first mode approaches zero. Furthermore, the same divergence of damping in the first two modes is observed as the flutter point is approached. Again, similar to the piston theory results, the damping and frequency of Modes 3 - 5 remains nearly constant with increasing Mach number. In order to asses the effect of grid resolution on the flutter boundary, these computations were repeated on a $0.27 \times 10^{6}$ cell grid. These results are shown in Fig. 27 , where $M_{f}=13.8\left(0.15<k_{\omega}<\right.$ 0.51). It is evident that coarsening the mesh by almost $60 \%$ does not significantly alter the flutter boundary at this altitude. However, it is important to note that as the flutter Mach number is increased, such as due to an increase in altitude, the shock will lie closer to the surface of the wing. Therefore, as the shock approaches the surface, grid resolution may become significant since the number of the points in the shock layer decreases. Note that the results generated using the $0.63 \times 10^{6}$ cell grid required 6.5 hours of CPU time using $12 \mathrm{AMD}$ Athlon 2000MP CPUs, where 500 time steps were used to generate 0.125 seconds of transient response. The ARMA system identification method was used to calculate the damping and frequency of the system.

Using the number of sub-iterations required for the Euler results as a guideline, the aeroelastic behavior of the low aspect ratio wing was determined for Navier-Stokes aerodynamics. For an initial estimate of the aeroelastic behavior using Navier-Stokes aerodynamics, $C F L_{\tau}=1.0$ with 50 sub-iterations was chosen. The results, shown in Fig. 28, are similar to those generated using third order piston theory and Euler aerodynamics. In this case, $M_{f}=13.6\left(0.14<k_{\omega}<0.48\right)$, which is only $1 \%$ lower than that predicted using Euler aerodynamics, and is $1.5 \%$ higher than that predicted using third order piston theory. As with 
the piston theory and Euler results, there is a coalescence in frequency, and a divergence in damping, of the first two modes, as the flutter Mach number is approached. Furthermore, the behavior of the higher modes is similar, where there is little change in the damping and frequency as the Mach number is increased. A notable difference between these results and the inviscid results is the gradual approach of the first mode to zero damping. Both the piston theory and Euler results had a more sudden change in damping at the flutter Mach number. Note that the Reynolds number for these computations is approximately $R e \approx 3 \times 10^{8}$ for the Mach numbers considered.

In order to asses the effect of $C F L_{\tau}$ on the flutter boundary, these computations were repeated using $C F L_{\tau}=5.0$. These results are shown in Fig. 29, where $M_{f}=13.65\left(0.14<k_{\omega}<0.50\right)$. The small change in the aeroelastic behavior and flutter Mach number due to the substantial increase in $C F L_{\tau}$ suggests that these results are converged to the correct solution. However, these aeroelastic results generated using NavierStokes aerodynamics should still be considered preliminary since the effect of sub-iterations has not been carefully studied. Also, it has been shown ${ }^{26}$ that grids on the order of $2 \times 10^{6}$ cells are required to converge the drag coefficient in steady-state computations. The effect of grid resolution on the hypersonic aeroelastic behavior of the wing is not known, and therefore needs further attention. These results appear reasonable, however, since it has been shown in Ref. 25 there are only small differences between the aeroelastic behavior of double-wedge typical section when either Euler or Navier-Stokes aerodynamics are used. Furthermore, note from Table 5 that the $y^{+}$values for the first gridpoint from the surface of the wing are $\mathcal{O}(1)$ for Mach numbers near the flutter boundary, implying that the grid resolution within the boundary layer may be adequate for these computations. These results were generated on a $0.63 \times 10^{6}$ cell grid, requiring 16.5 hours of CPU time using $12 \mathrm{AMD}$ Athlon 2000MP CPUs, where 500 time steps were used to generate 0.125 seconds of transient response. The ARMA system identification method was used to calculate damping and frequency of the system.

Note that the reduced frequencies, $k_{\omega}$, at flutter are small regardless of aerodynamic method used. This partially explains the good agreement between the Euler/Navier-Stokes results and the piston theory results. It is interesting to note that the aeroelastic behavior predicted using piston theory is conservative compared to the aeroelastic behavior predicted using Euler and Navier-Stokes aerodynamics. This is likely due to both three-dimensional flow effects which are not accounted for by piston theory, since it is strictly a point function relationship between the body and the pressure, and also, the small differences between the viscous and inviscid results for this configuration.

\section{Aerothermoelastic Behavior of a Three-Dimensional Low Aspect Ratio Wing}

The low aspect ratio cantilevered wing is a convenient example for studying the effects of aerodynamic heating on an aeroelastic system. For such a configuration the restrained warping at the root of the wing will induce thermal stresses which in turn will affect the torsional stiffness of the wing and modify its frequencies and mode shapes. The effect of warping restraint increases as the aspect ratio of a structure diminishes. ${ }^{64}$ Early studies of this effect ${ }^{65}$ indicate that by modeling a low aspect ratio wing as a plate, the effect of warping restraint is inherently included. More recently, this effect has been studied in the context of composites. ${ }^{66,67}$ In these studies, it has been pointed out that warping restraint is not only important for low-aspect ratio metallic structures, but also for composite structures where the material proprieties are non-isotropic. Furthermore, it was shown that the warping stiffness of a cantilever plate is a function of both aspect ratio, and the ratio of bending/torsion stiffness. ${ }^{1,66}$ This observation is important for hypersonic vehicles where structural properties are altered by aerodynamic heating.

In Ref. 26, it was determined that the low-aspect ratio wing was prone to thermal buckling due to local deformations along the leading and trailing edges. Therefore, the wing was modified for this study, in order to reduce the susceptibility to thermal buckling. Specifically, the leading and trailing edges have been stiffened, and several vertical spars have been added throughout the entire wing. This modification introduced minor changes in the structural mass and first two free vibration frequencies. The mass and vibration frequencies of the original and modified low aspect ratio wings are compared in Table 6 . The mode shapes of the modified wing are shown in Fig. 30. Note that only the fourth mode shape has changed significantly. For the original wing the fourth mode is an antisymmetric bending mode, and for the modified wing it is a symmetric bending mode.

Since the low aspect ratio wing is constructed of aluminum, it must be augmented with a thermal protection system (TPS) in order to provide reasonable heat transfer results in the high temperature environment of hypersonic flow. The TPS selected is based on Ref. 68. It consists of a $0.45 \mathrm{~mm}$ RENE' 41 metal heat 
shield, and a $3.8 \mathrm{~mm}$ flexible MIN-K thermal insulation blanket between the wing and heat shield. The RENE' 41 heat shield can withstand temperatures up to approximately $1500 \mathrm{~K}$, and is assumed to have an emissivity, $\epsilon=0.85$.

The normalized radiation equilibrium wall temperature for several Mach numbers and altitudes are shown in Fig. 31. The radiation equilibrium wall temperatures, $T_{R}$, were normalized by the peak equilibrium temperature, $\left(T_{R}\right)_{\max }$, which is listed below each case. These plots provide insight into the distribution in heating, as well as the magnitude of temperatures involved. In general, the heating is maximum at the leading edge, and minimum at the trailing edge. Also, note that as the Mach number is increased, the temperature distribution variation with chord increases. Furthermore, increasing the altitude increases the amount of chordwise variation in temperature. These plots illustrate that, even for a wing augmented with a radiative heat shield, the temperatures at equilibrium in hypersonic flow will surpass the melting temperature of aluminum $(\sim 900 \mathrm{~K})$ without active cooling. Furthermore, the temperatures present at $M=$ 10.0 , at $80,000 \mathrm{ft}$ and lower, will surpass the maximum temperature sustainable by the heat shield.

Figure 32 illustrates the effect of transient heating on the free vibration frequencies of the modified wing at $100,0000 \mathrm{ft}$ for $M=10.0,15.0$, and 20.0, at zero degree angle of attack. In each case, there is a reduction of the second, third, and fifth modal frequencies as the wing is heated. Also, there is a slight increase in the first frequency, and a more dramatic increase in the fourth modal frequency, which switches order with the fifth modal frequency. Also, in each case the wing buckles after a specific duration, namely 30 minutes, 18 minutes, and 13 minutes for $M=10.0, M=15.0$, and $M=20.0$ respectively. An interesting observation is the qualitative similarity of these three results. Each Mach number produces similar changes in frequency, with the largest difference in the results being the amount of time required for the changes to occur. This is likely due to increases in the heating rate due to increasing Mach number, while the heating distribution is relatively unchanged. In Fig. 33, the same results are presented; however, the frequencies are plotted as a function of a reference temperature, $T_{R e f}$. The reference temperature was chosen as the leading edge temperature of the $75 \%$ span location. Note that there are only minimal differences in the three cases when the results are plotted as a function of temperature. This plot illustrates that, at 100,000 ft, the wing is susceptible to buckling, for $660 \mathrm{~K}<T_{\text {Ref }}<680 \mathrm{~K}$, regardless of the Mach number. Furthermore, the free vibration characteristics of the heated structure at any $T_{R e f}$, can be estimated from one of the cases, implying that an analyst can reduce computation time required to calculate the effect of aerodynamic heating on the structure.

In practice, control surfaces on hypersonic vehicles will have a small angle of attack. It has been shown ${ }^{25}$ that, for the unheated case, a small angle of attack will not significantly alter the flutter boundary of a two dimensional typical section. When aerodynamic heating is considered, however, angle of attack is an important consideration since it introduces asymmetry in the temperature distribution between the upper and lower surfaces of a wing, and therefore introduces thermal stresses not present in zero degree angle of attack cases. The effect of angle of attack on the heated free vibration frequencies of the modified wing is shown in Fig. 34 at 100,000 ft for $\alpha_{s}=2^{\circ}$, and $M=20$. It is clear that, in the heated case, a small of angle of attack has a significant impact on the free vibration frequencies of the wing. Specifically, there is not a smooth change in frequency as the wing is heated, nor is there a clear distinction of where or if buckling occurs. Furthermore, the frequencies are much lower in the angle of attack case for $600 \mathrm{~K}<T_{R e f}<665 \mathrm{~K}$.

To gain insight into the effect of aerodynamic heating on the flutter boundary, the heated mode shapes of the wing were used to calculate the flutter boundary at 100,000 ft using third order piston theory aerodynamics. The heated configuration at $\alpha_{s}=2^{\circ}, T_{R e f}=665 \mathrm{~K}$ was chosen since it appears from Fig. 34 that the structure is significantly weakened at this point due to heating, yet is not buckled. The flutter boundary of the heated wing and cold wing are compared in Fig. 35. The ascent trajectory of the X-43, which is similar to the ascent trajectory of the now defunct NASP vehicle, is also included in order to illustrate the proximity of the wing flutter boundary to typical operating conditions. Note that due to the extremely high flutter boundary of the wing at the higher altitudes, the flutter boundary is also calculated using unsteady Newtonian impact aerodynamics, ${ }^{16,69}$ and used for comparison. At 100,000 ft there is a relatively small reduction in $M_{f}$ of the cold wing $\left(M_{f}=71.7\right)$ when a small amount of angle of attack $\left(M_{f}=62.6\right)$ is introduced. Furthermore, at 100,000 ft, $M_{f}$ is large implying that the piston theory results are inaccurate. However, they are conservative when compared to the Newtonian impact predictions, where $M_{f}=91.2$. It is evident from these results that neglecting aerodynamic heating results in large errors and a unrealistic prediction of the flutter boundary. With $\alpha_{s}=2^{\circ}$, comparing the heated wing, where $M_{f}=23.8$, to the cold wing, where $M_{f}=62.6$, the flutter boundary is reduced by $62 \%$ when aerodynamic heating is included. Also, 
the flutter boundary of the heated wing is $75 \%$ lower than the flutter boundary of the cold wing predicted using Newtonian impact aerodynamics. Based on these limited results it is evident that aerodynamic heating is an important consideration, however, this more complicated problem requires additional study.

\section{Aeroelastic Behavior of a Generic Reusable Launch Vehicle}

The model employed in this study is a vehicle that resembles a generic reusable launch vehicle. It consists of a lifting body and canted fins, and has some resemblance to the X-33 vehicle shown in Fig. 5. The dimensions of the generic vehicle are $76.2 \mathrm{ft}$. length, $45.54 \mathrm{ft}$. width, and $6 \mathrm{ft}$. thickness. The canted fins have a span of $18 \mathrm{ft}$. with a taper ratio of 0.25 . They have double-wedge cross-sections with the maximum thickness at mid-chord, equal to $3.33 \%$ of the chord. The empty mass of the vehicle is considered to be 70,000 lbs. The unrestrained modes were obtained using MSC.NASTRAN. The first five modes are depicted in Fig. 36. The first two modes are the symmetric and antisymmetric fin bending modes, while the third and fourth modes show fuselage bending and fuselage torsion. The fifth and higher modes show a combination of fin and fuselage deformation.

In previous hypersonic studies, the approximate aerodynamic method of choice for bluff bodies has been Newtonian impact theory blended with piston theory. ${ }^{16,69}$ The generic vehicle, shown in Fig. 5, has sharp leading edges. For $M>5$, the wedge angles are small enough to allow the presence of attached oblique shocks. However, the wedge angles are large and $M \tau \gg 1$, hence, instead of using a blended approach, a different approach, described next, is used.

The geometry of the generic hypersonic vehicle usually consists of a combination of flat surfaces having different wedge angles to the oncoming flow. In regions around the nose, and at the rear of the vehicle, the actual slope might be different due to localized curvature of the geometry. Since piston theory is a twodimensional theory which relates parameters in the $x-z$ plane, a sectional model of the vehicle can be created using piston theory alone. Fig. 37 shows that the streamlines along the surface in an inviscid flow have relatively small flow velocity components in the spanwise direction. Furthermore, at these Mach numbers, only a small portion at the root of the fin is affected by the flow around the fuselage (body). Therefore, the flow over the fins is assumed to be free-stream in this model. Thus, the surface of the vehicle can be divided into "flow zones", where the flow variables have substantially different values, due to the presence of oblique shocks and expansion fans. The local flow variables can thus be calculated at various points on the surface of the rigid geometry. Assuming small perturbations during the unsteady aeroelastic analysis, these values need not be recalculated, saving computational effort. Next, an unsteady aeroelastic analysis is carried out using piston theory with these local variables, applied in frames rotated to the angles of the flat surfaces approximating the fuselage.

Using third order piston theory, the aeroelastic behavior of the generic hypersonic vehicle was studied at different altitudes. The flutter Mach number, $M_{f}$, was calculated at 40,000 feet using piston theory with a varying number of structural modes. The results given in Table 7 indicate that the minimum number of modes required for a converged solution is 12 . Results obtained at 40,000 ft using piston theory, with 12 structural modes, are shown in Fig. 38, where $M_{f}=7.1$ and $k_{\omega}=0.3106$. In this case, flutter arises from the interaction among the higher modes, which have significant fin bending. The fuselage flexural and torsional modes (modes 3 and 4) do not play a significant role in this instability. Note, that tracking a substantial number of modes required a new graphical approach, ${ }^{44}$ as shown in Fig. 38. The upper half of the figure shows the variation in damping with Mach number, for the modes which are identified by a mode number that is specified along the third axis. The flutter Mach number is predicted when any of the damping curves rises above the shaded plane, which corresponds to zero damping. The lower half of the figure shows the variation of modal frequencies with Mach number for the various modes. The frequencies of the modes at flutter are indicated by black dots.

The values of the reduced frequencies for this case indicate that the use of a quasisteady approach such as piston theory is justified. Note that the reduced frequencies are calculated using the vehicle length as the reference length, and would be much smaller if the root chord length of the fin were used.

Figures 39 and 40 present the aeroelastic behavior of the generic vehicle using Euler aerodynamics. Figure 39 shows the aeroelastic behavior of the vehicle calculated on the coarse mesh, while Fig. 40 shows the aeroelastic behavior calculated on the refined mesh. Both series of simulations predict flutter at $M_{f}=9.3$, which is $31 \%$ higher than that predicted by nonlinear piston theory. The figures also show that all modes become equally unstable as the flutter boundary is reached, though the higher modes with significant fin bending are slightly less stable. The reduced frequency at flutter obtained on the coarse mesh was $k_{\omega}=0.1714$, 
while that on the refined mesh was $k_{\omega}=0.1626$.

The significant difference in flutter boundaries predicted using the two aerodynamic models is due to the presence of three-dimensional flow effects. Prior to calculation of the aeroelastic transients, the aeroelastic equilibrium state of the flexible vehicle was calculated by allowing the vehicle to deform in hypersonic flow, with artificial structural damping added to accelerate convergence. In the case of piston theory, which is a two-dimensional theory, as each cross-section of the vehicle is symmetrical about the horizontal plane, the equilibrium state of the flexible vehicle is the same as the rigid geometry at zero angle-of-attack. However, Euler aerodynamics is a fully three-dimensional aerodynamic model, and the cantedness of the fins leads to higher pressure on the upper surface compared to the lower surface, causing the fins to bend outwards along the span-wise direction. For inviscid flow with $M=8.0$ at 40,000 feet, the typical cross-section translates downward by nearly 1 foot. This deformation of the section is strongly dependent on the specific structural model of the fins and stiffeners.

Figure 41 depicts the typical cross-sections of a fin from rigid and aeroelastic equilibrium states in Euler flow, now superimposed. It is seen that the cross-section of the flexible vehicle has deformed and also developed a slight angle of attack. Carrying out a linear fit to the mean line of the cross-section in Euler flow indicates an effective angle of attack of $0.3^{\circ}$. However, Ref. 25 has indicated that introducing a small constant angle of attack in hypersonic flow, for the case of a two-dimensional double-wedge airfoil, produces only a slight change in the flutter boundary of the airfoil section. Figure 42 shows the surface pressures on the typical cross-sections of the rigid and flexible configurations at equilibrium in inviscid flow, and it is evident from the figure that these are quite different. Note that the surface pressures on the rigid vehicle using nonlinear piston theory is the same as that obtained using Euler aerodynamics. The deformation of the typical cross-section from the double-wedge airfoil leads to a significantly different pressure profile of the typical section. Thus, the introduction of flexibility interacts with the three-dimensional flow captured using Euler aerodynamics to change the equilibrium configuration of the generic vehicle. In comparison, the aeroelastic equilibrium state for the vehicle is the same as the undeformed state when using piston theory. Since the aeroelastic transients are calculated by considering small perturbations about the equilibrium position, the two models yield different aeroelastic behavior.

Figure 43 depicts the contours of $C_{p}$ around the typical section for the rigid vehicle at $M=8.6$ and 40,000 feet when using Euler aerodynamics, and Fig. 44 shows the flow around the deformed section when vehicle flexibility is introduced. Comparing the two figures shows the significant change in $C_{p}$ on and around the typical section. Similar differences were seen at $M=2.6$ at 10,000 feet and $M=25.0$ at 70,000 feet. As can be seen from these figures, the deformed section leads to an asymmetric distribution of pressure on the top and bottom surfaces, especially near the leading edge, unlike the rigid section which develops symmetric pressure loading on both surfaces.

For comparison, the aeroelastic simulations of the entire vehicle were also carried out using 7 modes. For this incomplete representation of the generic vehicle, both piston-theory and Euler-based aeroelastic studies indicate similar flutter behavior, with flutter arising from interaction between the symmetric fin bending mode and the fuselage flexural mode. This trend of good comparison between Euler aerodynamics and piston theory follows that also observed for the low aspect-ratio wing, and Ref. 19 for the X-43 geometry. For convenient comparison, the results of all the aeroelastic simulations described above are summarized in Table 8.

The flutter envelope for the generic vehicle, calculated using 12 modes, is shown in Fig. 45. This figure compares the flutter boundaries at various altitudes calculated using third-order piston theory, and Euler aerodynamics on the coarse and refined meshes. At low altitudes, nonlinear piston theory predicts flutter boundaries comparable with Euler aerodynamics-based methods for the generic vehicle. However, at altitudes above 30,000 feet, there is a significant difference, which becomes approximately constant above 50,000 feet. For the range between 10,000 and 50,000 feet, for which results could be obtained, the coarse mesh also compares well with the flutter results from the refined mesh.

The differences displayed in Fig. 45 are magnified in Fig. 46, which compares the dynamic pressure at flutter for the different altitudes considered. From this figure, it is obvious that the comparison between piston theory and Euler aerodynamics at low altitudes is not as good as seen in Fig. 45. At higher altitudes, piston theory underpredicts the flutter dynamic pressure compared to Euler aerodynamics by $25-50 \%$. However, piston theory does indicate a similar trend, including the maximum value corresponding to 70,000 feet. Considering the Euler results on the two meshes, good comparison is observed between 10,000 and 40,000 feet, while the results at 50,000 feet begin to show a divergence. Results at higher altitudes could not be 
obtained on the coarse mesh due to the insufficiently refined grid spacing near the surface, leading to a lack of convergence in the flow solutions. Solutions above 80,000 feet could not be obtained on the refined mesh due to a similar lack of convergence for $M>28$.

Another challenge with calculating flutter boundaries at very high altitudes is the length of the transient required to identify flutter. At altitudes below 70,000 feet, transients of 1 second have been used to identify flutter reliably with the MBA. At very high altitudes, due to the rarefied atmosphere, the aeroelastic loads are quite small even at high $M$. Figure 47 shows the aeroelastic transients for modes 1 and 9 , calculated at $M=28.0$ and 80,000 feet. The very gradual increase in the amplitude of mode 9 can be seen, although all the other modes were observed to be quite stable. For this flight condition, the computational resources required are an order of magnitude higher than that required for lower altitudes, as the transients have to be simulated over much longer periods. This is an excellent example of the importance of choosing an efficient time domain damping and frequency identification technique, such as the ARMA method.

The simulations in this study were carried out in parallel mode on nodes of a AMD cluster with 2000MP processors. For the time-step size selected, the run time to obtain an aeroelastic transient response of the generic vehicle at 40,000 feet using Euler aerodynamics was approximately 30 hours with 5 processors on the coarse mesh, and approximately 140 hours with 9 processors on the refined mesh. The use of either 7 or 12 structural modes did not significantly affect run time. A sample calculation carried out using Navier-Stokes aerodynamics required 36 days on 9 processors when using the refined mesh.

\section{Conclusions}

The fundamental studies in hypersonic aeroelasticity and aerothermoelasticity allow one to reach several useful conclusions.

1. In general, the three time domain frequency and damping identification techniques produce similar estimates of the aeroelastic behavior of a system. The ARMA method was superior, however, to both the LSCFM and MBA method since it provided quick damping and frequency estimates with minimal response record length. This is an important consideration because the cost of computational aeroelastic simulations is largely determined by the length of response record required to accurately calculate system damping. In this case, the ARMA method offers a $75 \%$ reduction in computational cost over the MBA.

2. The aeroelastic behavior of a system, predicted using time-accurate CFD solutions of the Euler equations are sensitive to the number of sub-iterations used, as well as $C F L_{\tau}$. Studies must be carried out on a case by case basis in order to ensure that these parameters have appropriate values.

3. The aeroelastic behavior of the three-dimensional low aspect ratio wing obtained using piston theory, Euler and Navier-Stokes aerodynamics is similar. The flutter boudary obtained using Euler aerodynamics is approximately $2 \%$ higher than that predicted by piston theory, while the flutter boundary obtained using Navier-Stokes aerodynamics is less than 1\% lower than the Euler solution.

4. The presence of aerodynamic heating on a low aspect ratio cantilever structure, such as a fin and/or control surface on a hypersonic vehicle, will result in thermal stresses due to warping restraint at the root. This, combined with material property degradation, dramatically affects the natural frequencies of the structure. Increasing the Mach number decreases the time before thermal buckling occurs. However, changes in frequency are similar for various Mach numbers when plotted as a function of leading edge temperature.

5. Angle of attack is an important consideration when performing aerothermoelastic analysis of a structure, since it introduces additional thermal stresses that significantly degrade the stiffness of the structure for a given reference temperature, or point in time. Aerodynamic heating in this case has a substantial effect on the aeroelastic behavior of the low aspect ratio wing, reducing the flutter boundary by $62 \%$.

6. For the generic vehicle, the aeroelastic model based on Euler solutions predicts a flutter boundary $31 \%$ higher than that predicted by third-order piston theory, due to the presence of significant threedimensional flow effects captured by Euler aerodynamics. This leads to significant deformation of 
the canted fins, and different equilibrium configurations for the flexible vehicle are predicted by these two aerodynamic models. Hence, the surface pressure distributions are also found to be different. Since the aeroelastic transient solutions are calculated by considering small perturbations about these equilibrium positions, different aeroelastic behavior is predicted by these models.

7. Based on the various aeroelastic simulations described in this study, it can be seen that the differences between approximate solutions based on third-order piston theory and more exact solutions based on Euler and Navier-Stokes aerodynamics can be small, or large, and these differences depend on the vehicle configuration and flight conditions.

8. The relatively low reduced frequencies observed for both the low aspect ratio wing and the generic hypersonic vehicle can be used to justify the quasisteady nature of piston theory loads. Also, such an approximation can also be used to increase computational efficiency when carrying out Euler or Navier-Stokes computations.

9. The aeroelastic behavior of the configurations considered is relatively insensitive to grid resolution as long as a sufficient number of grid points are within the shock layer of the flow.

10. The results presented can be considered to provide a partial validation of the aeroelastic capabilities of the CFL3D code for the hypersonic flow regime.

\section{Acknowledgments}

The authors wish to express their gratitude to NASA Langley Research Center for the CFL3D code and thank Dr. R. Biedron for his help in using this code. This research was partially funded by the Space Vehicle Technology Institute under grant NCC3-989 jointly funded by NASA and DOD within the NASA Constellation University Institutes Project, with Claudia Meyer as the project manager; and Air Force Grant F49620-01-0158 with Dr. Clark Allred as program manager.

\section{References}

\footnotetext{
${ }^{1}$ Bisplinghoff, R.L. and Dugundji, J., Influence of Aerodynamic Heating on Aeroelastic Phenomena, Pergamon Press, 1958, pp. 288-312.

${ }^{2}$ Garrick, I.E., "A Survey of Aerothermoelasticity," Aerospace Engineering, January, 1963, pp. 140-147.

${ }^{3}$ Hedgepeth, J. and Widmayer, E., "Dynamic and Aeroelastic Problems of LIfting Re-Entry Bodies," Aerospace Engineering, January, 1963, pp. 148-153.

${ }^{4}$ Laidlaw, W.R. and Wyker, J.H., "Potential Aerothermoelastic Problems Associated with Advanced Vehicle Design," Aerospace Engineering, January, 1963, pp. 154-164.

${ }^{5}$ Xue, D.Y. and Mei, C., "Finite Element Two-Dimensional Panel Flutter at High Supersonic Speeds and Elevated Temperature," Proc. 31st AIAA/ASME/ASCE/AHS/ASC Structures, Structural Dynamics and Materials Conference, No. AIAA Paper No. 90-0982, 1990, pp. 1464-1475.

${ }^{6}$ Gray, E.G. and Mei, C., "Large-Amplitude Finite Element Flutter Analysis of Composite Panels in Hypersonic Flow," Proc. 33rd AIAA/ASME/ASCE/AHS/ASC Structures, Structural Dynamics and Materials Conference, No. AIAA Paper No. 92-2130, Dallas, TX, April 16-17 1992, pp. 492-512.

${ }^{7}$ Abbas, J.F. and Ibrahim,R.A., "Nonlinear Flutter of Orthotropic Composite Panel Under Aerodynamic Heating," AIAA J., Vol. 31, No. 8, 1993, pp. 1478-1488.

${ }^{8}$ Bein, T., Friedmann, P., Zhong, X., and Nydick, I., "Hypersonic Flutter of a Curved Shallow Panel with Aerodynamic Heating," Proc. 34th AIAA/ASME/ASCE/AHS/ASC Structures, Structural Dynamics and Materials Conference, No. AIAA Paper No. 93-1318, La Jolla, CA, April 19-22 1993.

${ }^{9}$ Nydick, I., Friedmann, P.P., and Zhong, X., "Hypersonic Panel Flutter Studies on Curved Panels," Proc. 36th AIAA/ASME/ASCE/AHS/ASC Structures, Structural Dynamics and Materials Conference, No. AIAA Paper no. 95-1485, New Orleans, LA, April 1995, pp. 2995-3011.

${ }^{10}$ Mei, C., Abdel-Motagly, K., and Chen, R., "Review of Nonlinear Panel Flutter at Supersonic and Hypersonic Speeds," Applied Mechanics Reviews, 1998.

${ }^{11}$ Ricketts, R., Noll, T., Whitlow, W., and Huttsell,L., "An Overview of Aeroelasticity Studies for the National Aerospace Plane," Proc. 34th AIAA/ASME/ASCE/AHS/ASC Structures, Structural Dynamics and Materials Conference, No. AIAA Paper No. 93-1313, La Jolla, CA, April 19-22 1993, pp. 152 - 162.

${ }^{12}$ Spain, C., Zeiler, T.A., Bullock, E., and Hodge, J.S., "A Flutter Investigation of All-Moveable NASP-Like Wings at Hypersonic Speeds," Proc. 34th AIAA/ASME/ASCE/AHS/ ASC Structures, Structural Dynamics and Materials Conference, No. AIAA Paper No. 93-1315, La Jolla, CA, April 19-22 1993.
} 
${ }^{13}$ Scott, R.C. and Pototzky, A.S., "A Method of Predicting Quasi-Steady Aerodynamics for Flutter Analysis of High Speed Vehicles Using Steady CFD Calculations," Proc. 34th AIAA/ASME/ASCE/AHS/ASC Structures, Structural Dynamics and Materials Conference, No. AIAA Paper No. 93-1364, La Jolla, CA, April 19-22 1993, pp. 595-603.

${ }^{14}$ Spain, C., Zeiler, T.A., Gibbons, M.D., Soistmann, D.L., Pozefsky, P., DeJesus, R.O., and Brannon,C.P., "Aeroelastic Character of a National Aerospace Plane Demonstrator Concept," Proc. 34th AIAA/ASME/ASCE/AHS/ ASC Structures, Structural Dynamics and Materials Conference, La Jolla, CA, April 19-22 1993, pp. 163-170.

${ }^{15}$ Rodgers, J.P., "Aerothermoelastic Analysis of a NASP-Like Vertical Fin," Proc. 33rd AIAA/ASME/ASCE/AHS Structures, Structural Dynamics and Materials Conference, No. AIAA-92-2400-CP, Dallas, TX, April 1992.

${ }^{16}$ Heeg, J., Zeiler, T., Pototzky, A., Spain, C., and Engelund, W., "Aerothermoelastic Analysis of a NASP Demonstrator Model," Proc. 34th AIAA/ASME/ASCE/AHS/ ASC Structures, Structural Dynamics and Materials Conference, No. AIAA Paper No. 93-1366, La Jolla, CA, April 19-22 1993, pp. 617-627.

${ }^{17}$ Heeg, J. and Gilbert, M.G., "Active Control of Aerothermoelastic Effects for a Conceptual Hypersonic Aircraft," Journal of Aircraft, Vol. 30, 1993, pp. 453-458.

${ }^{18}$ Blades, E., Ruth, M., and Fuhrman, D., "Aeroelastic Analysis of the X-34 Launch Vehicle," Proc. 40th AIAA/ASME/ASCE/AHS/ ASC Structures, Structural Dynamics and Materials Conference, No. AIAA Paper No. 99-1352, St. Louis, MO, 1999, pp. 1321-1331.

${ }^{19}$ Gupta, K.K., Voelker, L.S., Bach, C., Doyle, T., and Hahn, E., "CFD-Based Aeroelastic Analysis of the X-43 Hypersonic Flight Vehicle," 39th Aerospace Sciences Meeting \&s Exhibit, No. AIAA Paper No. 2001-0712, 2001.

${ }^{20}$ Nydick, I. and Friedmann, P.P., "Aeroelastic Analysis of a Generic Hypersonic Vehicle," Proc. CEAS/AIAA/ICASE/NASA Langley International Forum on Aeroelasticity and Structural Dynamics, No. NASA/CP1999-209136/PT2, Williamsburg, VA, June 22-25 1999, pp. 777-810.

${ }^{21}$ Friedmann, P.P., McNamara, J.J., Thuruthimattam, B.J., and Nydick, I., "Aeroelastic Analysis of Hypersonic Vehicles," J. of Fluids and Structures, Vol. 19, 2004, pp. 681-712.

${ }^{22}$ Berry, S.A., Horvath, T.J., Hollis, B.R., Thompson, R.A., and Hamilton, H.H., "X-33 Hypersonic Boundary Layer Transition," 33rd AIAA Thermophysics Conference, No. AIAA Paper No. 99-3560, Norfolk, VA, June 28 - July 11999.

${ }^{23}$ Riley, C.J., Kleb, W.L., and Alter, S.J., "Aeroheating Predictions for X-34 Using An Inviscid-Boundary Layer Method," 36th Aerospace Sciences Meeting \& Exhibit, No. AIAA 98-0880, Reno, NV, January 1998.

${ }^{24}$ Thuruthimattam, B.J., Friedmann, P.P., McNamara, J.J., and Powell, K.G., "Aeroelasticity of a Generic Hypersonic Vehicle," Proc. 43rd AIAA/ASME/ASCE/AHS Structures, Structural Dynamics and Materials Conference, No. AIAA Paper No. 2002-1209, Denver, CO, April 2002.

${ }^{25}$ Thuruthimattam, B.J., Friedmann, P.P., McNamara, J.J., and Powell, K.G., "Modeling Approaches to Hypersonic Aerothermoelasticity with Application to Resuable Launch Vehicles," Proc. 44th AIAA/ASME/ASCE/AHS Structures, Structural Dynamics and Materials Conference, No. AIAA Paper No. 2003-1967, Norfolk, VA, April 2003.

${ }^{26}$ McNamara, J.J., Thuruthimattam, B.J., Friedmann, P.P., Powell, K.G., and Bartels, R.E., "Hypersonic Aerothermoelastic Studies for Reusable Launch Vehicles," Proc. 45th AIAA/ASME/ASCE/AHS Structures, Structural Dynamics and Materials Conference, No. AIAA Paper No. 2004-1590, Palm Springs, CA, April 2004.

${ }^{27}$ Krist, S.L., Biedron, R.T., and Rumsey, C.L., CFL3D User's Manual (Version 5.0), NASA, TM 1998-208444, 1997, CFL3D v.6 update:

http://cfl3d.larc.nasa.gov/Cf13dv6/cfl3dv6.html.

${ }^{28}$ Bartels, R.E., "Mesh Strategies for Accurate Computation of Unsteady Spoiler and Aeroelastic Problems," Journal of Aircraft, Vol. 37, No. 3, 2000, pp. 521-525.

${ }^{29}$ Robinson, B.A., Batina, J.T., and Yang, H.T., "Aeroelastic Analysis of Wings Using the Euler Equations with a Deforming Mesh," Journal of Aircraft, Vol. 28, November 1991, pp. 778-788.

${ }^{30}$ Cunningham, H.J., Batina, J.T., and Bennett, R.M., "Modern Wing Flutter Analysis by Computational Fluid Dynamic Methods," Journal of Aircraft, Vol. 25, No. 10, 1989, pp. 962-968.

${ }^{31}$ Lee-Rausch, E.M. and Batina, J.T., "Wing Flutter Boundary Prediction Using Unsteady Euler Aerodynamic Method," Proc. 34th AIAA/ASME/ASCE/AHS Structures, Structural Dynamics and Materials Conference, No. AIAA Paper No. 931422, 1993, pp. 1019-1029.

${ }^{32}$ Lee-Rausch, E.M. and Batina, J.T., "Calculation of AGARD Wing 445.6 Flutter Using Navier-Stokes Aerodynamics," Proc. AIAA 11th Applied Aerodynamics Conference, No. AIAA Paper No. 93-3476, Hampton, VA, August 9-11 1993.

${ }^{33}$ Silva, W. and Bartels, R., "Development of Reduced-Order Models for Aeroelastic Analysis and Flutter Prediction Using the CFL3Dv6.0 code," Journal of Fluids and Structures, Vol. 19, 2004, pp. 729-745.

${ }^{34}$ Batina, J.T., "Unsteady Euler Airfoil Solutions Using Unstructured Dynamic Meshes," AIAA Journal, Vol. 28, 1990, pp. 1381-1388.

${ }^{35}$ Tezduyar, T.E., Behr, M., and Liou, J., "A New Strategy for Finite Element Computations involving Moving Boundaries and Interfaces - The Deforming-spatial-domain/Space-time Procedure: I. The concept and the Preliminary Numerical Tests," Computer Methods in Applied Mechanics and Engineering, Vol. 94, 1992, pp. 339-351.

${ }^{36}$ Hughes, T.J.R. and Hulbert, G.M., "Space-Time Finite Element Methods For Elastodynamics: Formulations and Error Estimates," Computer Methods in Applied Mechanics and Engineering, Vol. 66, 1988, pp. 339-363.

${ }^{37}$ Patil, M.J., Hodges, D.H., and Cesnik, C.E.S., "Nonlinear Aeroelastic Analysis of Complete Aircraft in Subsonic Flow," Journal of Aircraft, Vol. 37, No. 5, September 2000, pp. 753-760.

${ }^{38}$ Donea, J., Guiliani, S., and Halleux, J.P., "An Arbitrary Lagrangian-Eulerian Finite Element Method for Transient Dynamic Fluid-Structure Interactions," Computer Methods in Applied Mechanics and Engineering, Vol. 33, 1982, pp. 689-723.

${ }^{39}$ Bendiksen, O.O., "A New Approach to Computational Aeroelasticity," Proc. AIAA/ASME/ASCE/AHS/ASC 32nd Structure, Structural Dynamics and Materials Conf., Baltimore, MD, April 8-9 1991, pp. 1712-1727, AIAA Paper 91-0939. 
${ }^{40}$ Farhat, C., Lesoinne, M., and Maman, N., "Mixed Explicit/Implicit Time Integration of Coupled Aeroelastic Problems: Three-field Formulation, Geometric Conservation and Distributed Solution," International Journal for Numerical Methods in Fluids, Vol. 21, 1995, pp. 807-835.

${ }^{41}$ Tran H. and Farhat, C., "An Integrated Platform for the Simulation of Fluid-Structure-Thermal Interaction Problems," Proc. 43rd AIAA/ASME/ASCE/AHS Structures, Structural Dynamics and Materials Conference, No. AIAA Paper No. 20021307, Denver, CO, April 2002.

${ }^{42}$ Stephens, C.H., Arena Jr., A.S., and Gupta, K.K., "Application of the transpiration method for aeroservoelastic prediction using CFD," Proceedings of the 39th AIAA/ASME/ASCE/AHS/ASC Structures, Structural Dynamics, and Materials Conference and Exhibit, No. AIAA Paper 98-2071, Long Beach, CA, April 20-23 1998.

${ }^{43}$ Hartwich, P. and Agrawal, S., "Perturbing Multiblock Patched Grids in Aeroelastic Design Optimization Applications," No. AIAA 97-2038, June 1997.

${ }^{44}$ Thuruthimattam, B.J., Fundamental Studies in Hypersonic Aeroelasticity Using Computational Methods, Ph.D. thesis, The University of Michigan, 2005.

${ }^{45}$ Bartels, R.E., "New Mesh Movement and Aeroelasticity in CFL3D: Implementation and Use," NASA TM to be published, 2005.

${ }^{46}$ Bousman, W.G. and Winkler, D.J., "Application of the Moving-Block Analysis," Proceedings of the AIAA Dynamics Specialist Conference, No. AIAA 81-0653, Atlanta, GA, April 1981, pp. 755-763.

${ }^{47}$ Hammond, C.E. and Dogget, Jr. R.V., "Determination of Subcritical Damping by Moving-Block/Randomdec Applications," Proceedings of the Conference on Flutter Testing Techniques, NASA Dryden RC, Edwards, CA, October 1975, pp. $59-76$.

${ }^{48}$ Bennett, R.G. and Desmarais, R., "Curve Fitting of Aeroelastic Transient Response Data with Exponential Functions," No. NASA-SP-415, Oct. 1975, pp. 43-58.

${ }^{49}$ Matsuzaki, Y. and Ando, Y., "Estimation of Flutter Boundary from Random Responses due to Turbulence at Subcritical Speeds," J. of Aircraft, Vol. 18, No. 10, 1981, pp. 862-868.

${ }^{50}$ Pak, C.G. and Friedmann, P.P., "New Time Domain Technique for Flutter Boundary Identification," Proceedings of the AIAA Dynamics Specialist Conference, No. AIAA Paper 92-2102, Dallas, Tx, April 1992, pp. 201-214.

${ }^{51}$ Batill, S.M., Carey, D.M., and Kehoe, M.W., "Digital Time Series Analysis for Flutter Test Data," Proceedings of the AIAA Dynamics Specialist Conference, No. AIAA Paper 92-2103, Dallas, Tx, April 1992, pp. 215-223.

${ }^{52}$ Chen, P.C. and Liu, D.D., "Unified Hypersonic/Supersonic Panel Method for Aeroelastic Applications to Arbitrary Bodies," Journal of Aircraft, Vol. 39, No. 3, 2002, pp. 499-506.

${ }^{53}$ Liu, D.D., Chen, P.C., Tang, L., Chang, K.T., and Gao, X.W., "Expedient Hypersonic Aerothermodynamics Methodology for RLV/TPS Design," Proc. 11th AIAA/AAAF International Conference on Space Planes and Hypersonic Systems and Technologies, Orleans, France, September 2002.

${ }^{54}$ Ashley, H. and Zartarian, G., "Piston Theory - A New Aerodynamic Tool for the Aeroelastician," Journal of the Aeronautical Sciences, Vol. 23, No. 12, 1956, pp. 1109-1118.

${ }^{55}$ Lighthill, M.J., "Oscillating Airfoils at High Mach Numbers," Journal of the Aeronautical Sciences, Vol. 20, No. 6, June 1953.

${ }^{56}$ Anderson, J.D., Aerothermodynamics: A Tutorial Discussion, chap. 1, AIAA, 1992, pp. 3-57.

${ }^{57}$ Bisplinghoff, R.L., "Some Structural and Aeroelastic Considerations of High-Speed Flight," Journal of the Aerospace Sciences, Vol. 23, No. 4, April 1956, pp. 289-329.

${ }^{58}$ Rogers, M., "Aerothermoelasticity," AeroSpace Engineering, October 1958, pp. 34-43.

${ }^{59}$ Garrick, I.E., "Aeroelasticity Effects of High Temperatures," Aerospace Engineering, January 1963, pp. 140-147.

${ }^{60}$ Budiansky, B. and Mayers, J., "Influence of Aerodynamic Heating on the Effective Torsional Stiffness of Thin Wings," Journal of the Aeronautical Sciences, December 1956, pp. 1081-1093.

${ }^{61}$ Bertin, J.J., Hypersonic Aerothermodynamics, AIAA, Washington, DC, 1994.

${ }^{62}$ Thornton, E.A., Thermal Structures for Aerospace Applications, AIAA, Reston, VA, 1996.

63 "MSC.NASTRAN Reference Manual," 2001.

${ }^{64}$ Bisplinghoff, R.L., Ashley, H., and Halfman, R.L., Aeroelasticity, Addison-Wesley, 1955.

${ }^{65}$ Reissner, E. and Stein M., "Torsion and Transverse Bending of Cantilever Plates," NACA TN 2369, 1951.

${ }^{66}$ Crawley, E.F. and Dugundji, J., "Frequency Determination and Non-Dimensionalization for Composite Cantilever Plates," Journal of Sound and Vibration, Vol. 72, No. 1, 1980, pp. 1-10.

${ }^{67}$ Gern, F. and Librescu, L., "Aeroelastic Tailoring of Composite Wings Exhibiting Nonclassical Effects and Carrying External Stores," Journal of Aircraft, Vol. 37, No. 6, 2000, pp. 1097-1104.

${ }^{68}$ Ellis, D.A., Pagel, L.L., and Schaeffer, D.M., "Design and Fabrication of a Radiative Actively Cooled Honeycomb Sandwich Structural Panel for a Hypersonic Aircraft," NASA CR 2957, 1978.

${ }^{69}$ Nydick, I., Studies in Hypersonic Aeroelasticity, Ph.D. thesis, University of California, Los Angeles, 2000. 
Table 1. Error in moment calculation for typical section as a function of grid spacing normal to the vehicle surface, nondimensionalized by fuselage length.

\begin{tabular}{|c|c|c|}
\hline Nondimensional grid-spacing $\left(\Delta_{k}\right)$ & $C_{M}$ & Error $(\%)$ \\
\hline $2 \times 10^{-6}$ & -0.1281 & 0.2799 \\
$5 \times 10^{-7}$ & -0.1279 & 0.1347 \\
$1 \times 10^{-8}$ & -0.1278 & 0.0490 \\
$1 \times 10^{-9}$ & -0.1277 & $\mathrm{~N} / \mathrm{A}$ \\
\hline
\end{tabular}

Table 2. Parameters describing the double-wedge airfoil.

\begin{tabular}{|c|c|}
\hline Parameter & \\
\hline$c(\mathrm{~m})$ & 2.35 \\
Thickness ratio $(\%)$ & 3.36 \\
$m(\mathrm{~kg} / \mathrm{m})$ & 94.2 \\
$r_{\alpha}$ & 0.484 \\
$\omega_{h}(\mathrm{~Hz})$ & 13.4 \\
$\omega_{\alpha}(\mathrm{Hz})$ & 37.6 \\
$x_{\alpha}$ & 0.2 \\
$a$ & 0.1 \\
\hline
\end{tabular}

Table 3. Comparison of the Lockheed F-104 Starfighter wing to the low aspect ratio wing model

\begin{tabular}{|c|c|c|}
\hline Parameter & F-104 & 3-D Wing \\
\hline \hline Wing Mass (Kg) & 350.28 & 350.05 \\
1st Bending Frequency (Hz) & 13.40 & 13.41 \\
1st Torsional Frequency (Hz) & 37.60 & 37.51 \\
\hline
\end{tabular}

Table 4. Flutter results for the low aspect ratio wing using an increasing number of modes, and third order piston theory aerodynamics, at $40,000 \mathrm{ft}$.

\begin{tabular}{|c|c|}
\hline No. of Modes & $M_{f}$ \\
\hline \hline 2 & 15.6 \\
5 & 13.4 \\
8 & 13.3 \\
\hline
\end{tabular}


Table 5. Average $y^{+}$values for the first gridpoint from the wing surface at Mach numbers near the flutter boundary $\left(M_{f}=13.65\right.$ at $\left.\mathbf{4 0 , 0 0 0} \mathrm{ft}\right)$ obtained using Navier-Stokes aerodynamics.

\begin{tabular}{|c|c|}
\hline Avg. $y^{+}$ & $M$ \\
\hline \hline 2.50 & 12.0 \\
2.37 & 12.5 \\
2.34 & 13.0 \\
2.30 & 13.5 \\
2.27 & 14.0 \\
\hline
\end{tabular}

Table 6. Comparison of the original and modified low aspect ratio wings

\begin{tabular}{|c|c|c|}
\hline Parameter & Original Wing & Modified Wing \\
\hline \hline Wing Mass (Kg) & 350.05 & 377.73 \\
Mode 1 Freq. (Hz) & 13.41 & 14.28 \\
Mode 2 Freq. (Hz) & 37.51 & 40.94 \\
Mode 3 Freq. (Hz) & 49.18 & 60.06 \\
Mode 4 Freq. (Hz) & 77.14 & 81.86 \\
Mode 5 Freq. (Hz) & 79.48 & 97.25 \\
\hline
\end{tabular}

Table 7. Flutter results for the generic vehicle using an increasing number of modes, and third order piston theory aerodynamics, at $40,000 \mathrm{ft}$.

\begin{tabular}{|c|c|}
\hline No. of modes & $M_{f}$ \\
\hline \hline 7 & 11.6 \\
10 & 7.8 \\
12 & 7.1 \\
14 & 7.1 \\
\hline
\end{tabular}

Table 8. Comparison of flutter boundaries for the generic vehicle at 40,000 feet, obtained using different models for predicting aerodynamic loads.

\begin{tabular}{|c|c|c|c|c|}
\hline \multirow{2}{*}{$\begin{array}{c}\text { Aerodynamic } \\
\text { model }\end{array}$} & \multicolumn{2}{|c|}{7 modes } & \multicolumn{2}{c|}{12 modes } \\
\cline { 2 - 5 } & $M_{f}$ & $k_{\omega}$ & $M_{f}$ & $k_{\omega}$ \\
\hline Piston theory, nonlinear & 11.7 & 0.1344 & 7.1 & 0.3106 \\
Euler, coarse mesh & 11.6 & 0.1412 & 9.3 & 0.1714 \\
Euler, refined mesh & 11.2 & 0.1426 & 9.3 & 0.1626 \\
\hline
\end{tabular}




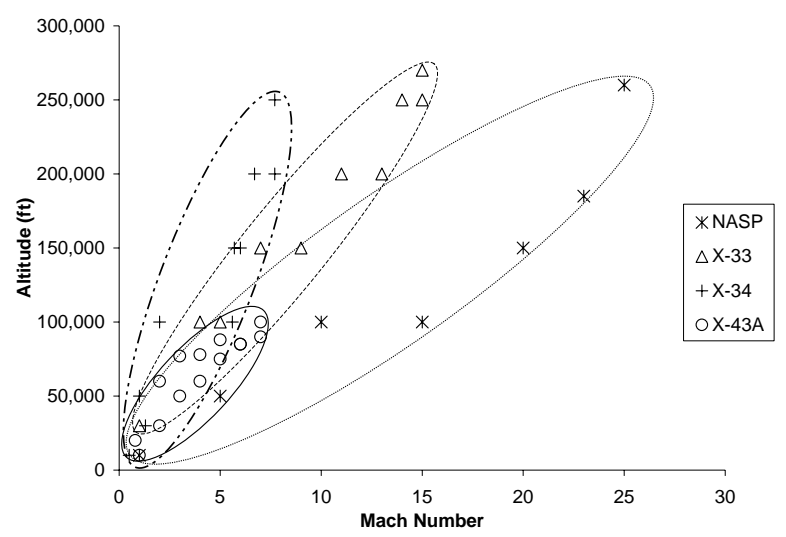

Figure 1. Operating envelopes for several modern hypersonic vehicles.

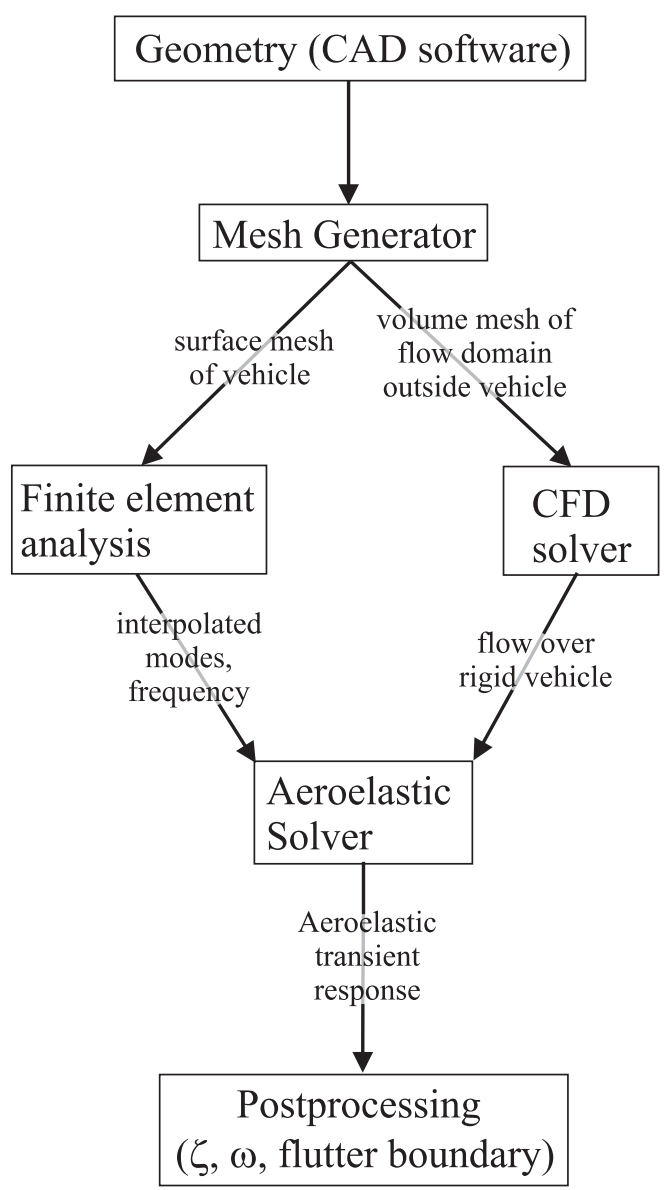

Figure 2. A flow diagram of the computational aeroelastic solution procedure. 


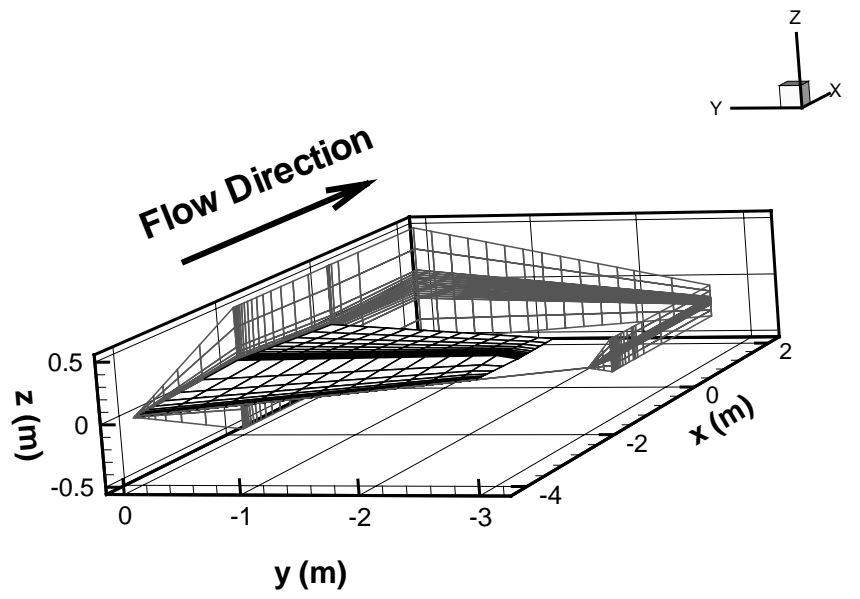

Figure 3. Computational domain of the low aspect ratio wing.

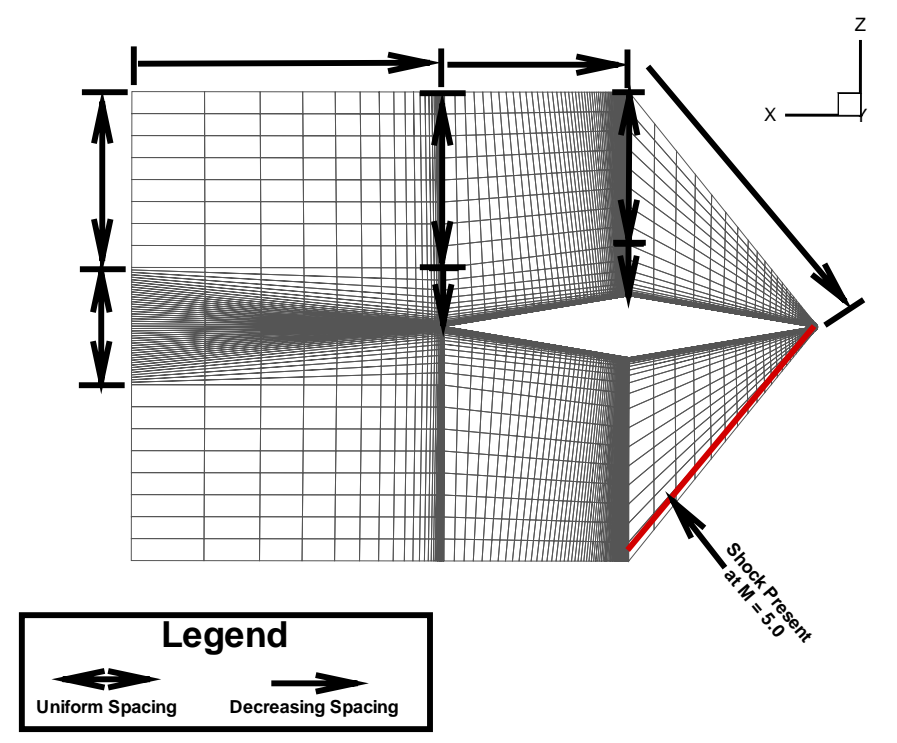

Figure 4. Span section plane of the computational domain at the root of the low aspect ratio wing. Note that the $\mathrm{z}$ dimension is scaled relative to the $\mathrm{x}$ and $\mathrm{y}$ dimensions for visualization purposes. 


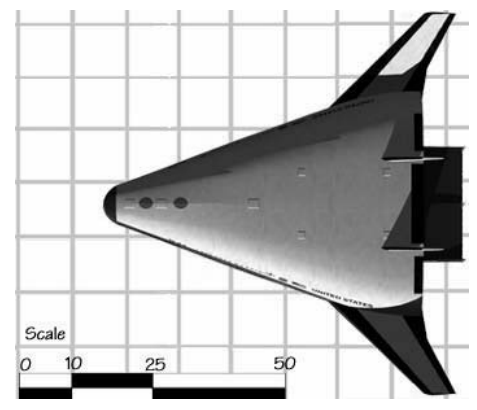

(a) X33, top view.

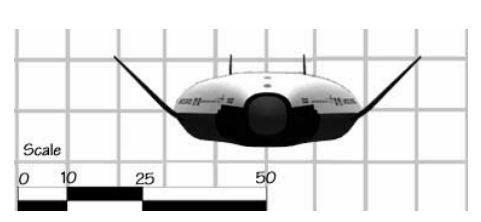

(c) X33, front view.

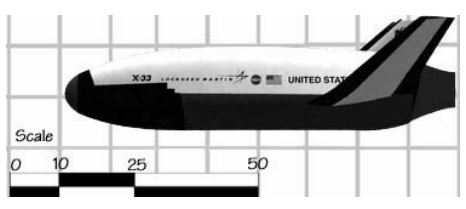

(e) X33, side view.

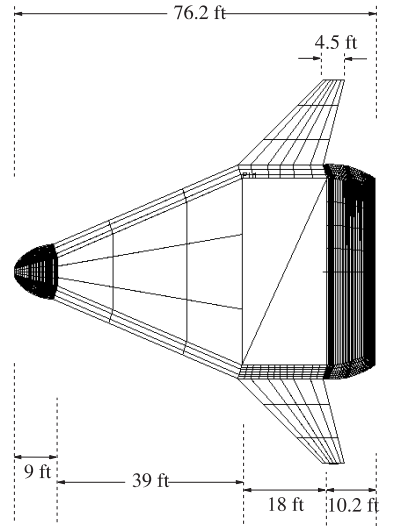

(b) Generic vehicle, top view.

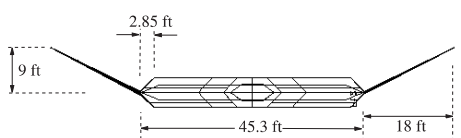

(d) Generic vehicle, front view.

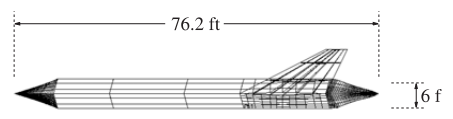

(f) Generic vehicle, side view.

Figure 5. X-33 and generic reusable launch vehicle.

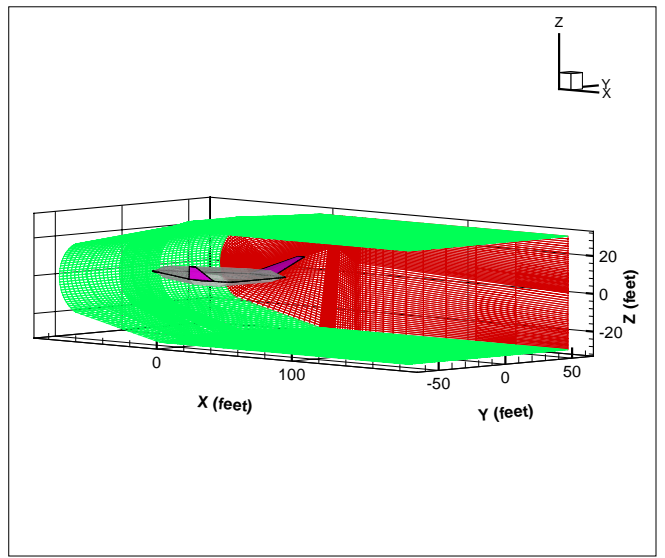

Figure 6. CFD domain for aeroelastic analysis of the generic hypersonic vehicle. 


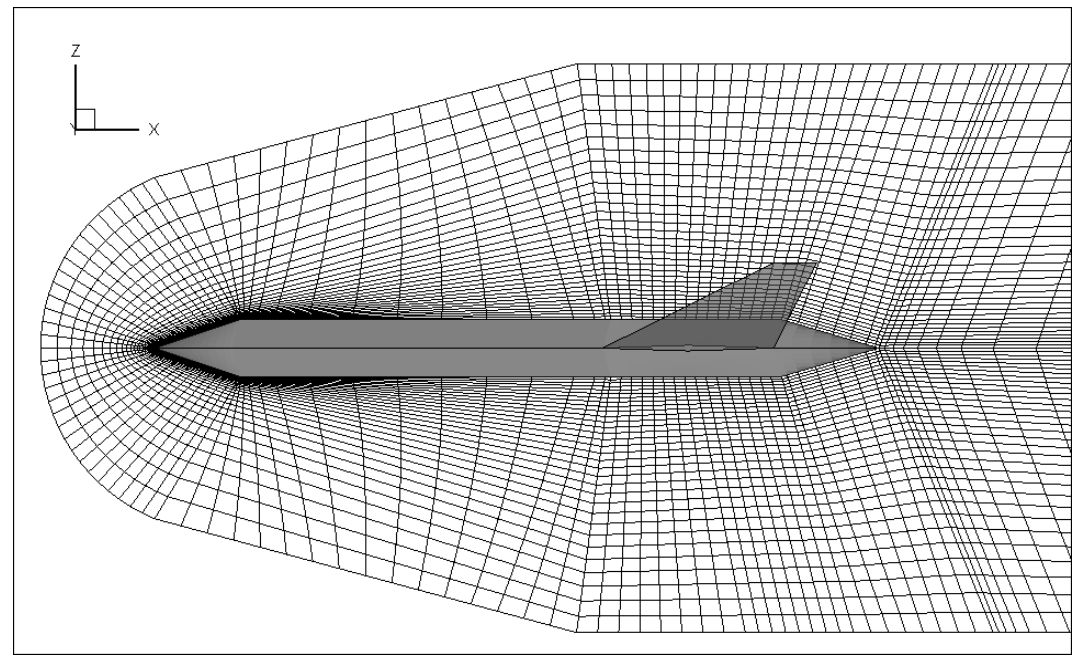

Figure 7. A view of the midplane of the grid around the generic vehicle.

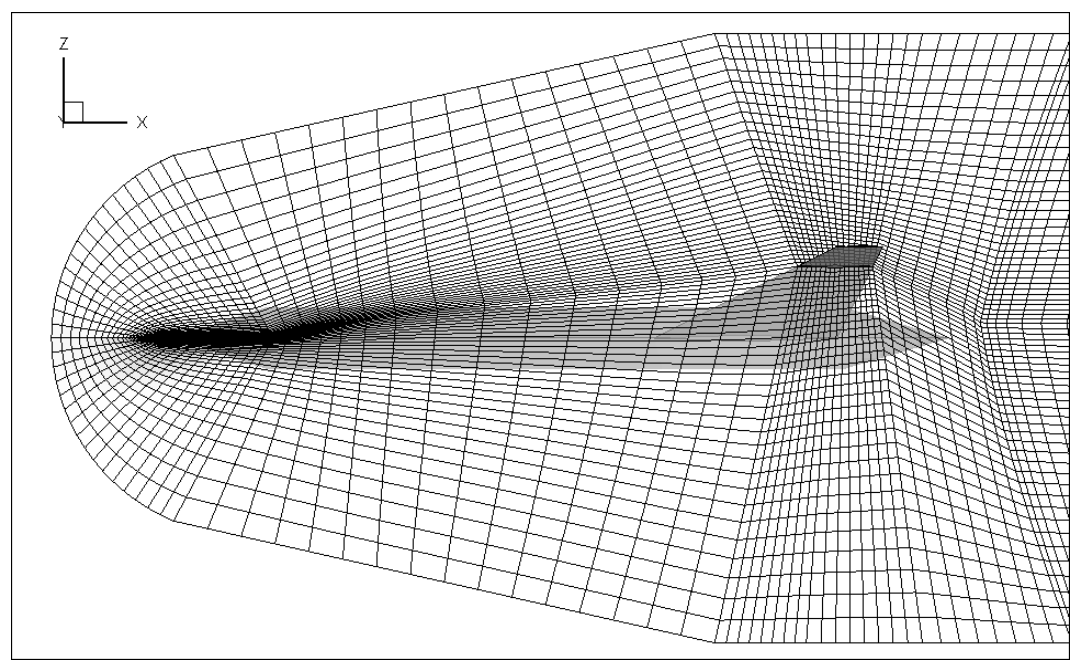

Figure 8. A view of the grid around the typical cross-section of the generic vehicle.

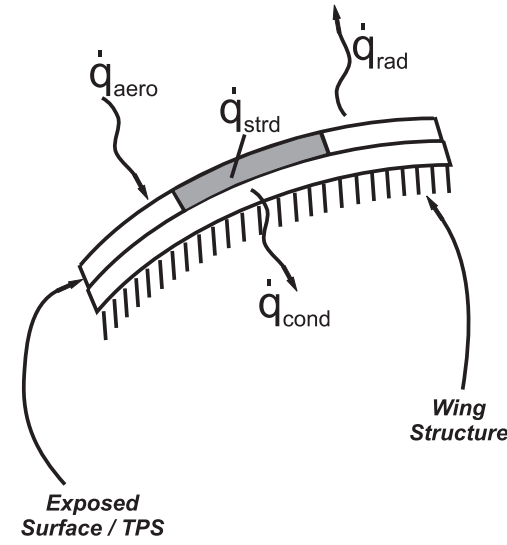

Figure 9. Heat transfer at the wall of a hypersonic vehicle. 


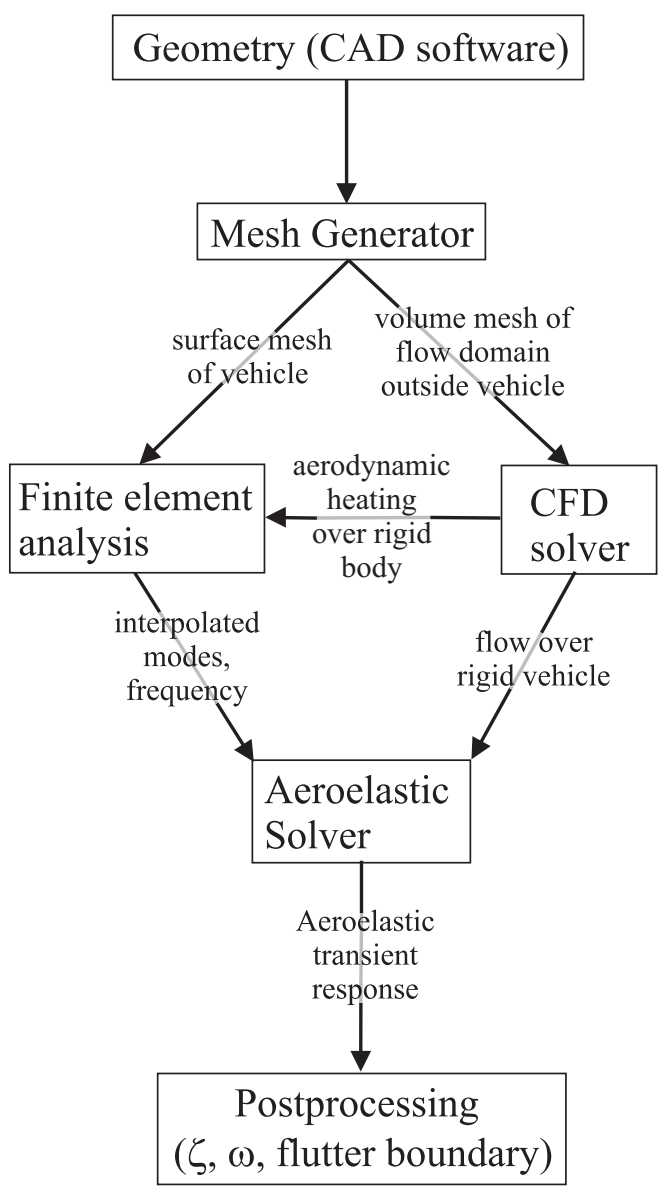

Figure 10. A flow diagram for the computational aerothermoelastic solution procedure. The aerodynamic heating information is passed from the CFD solver to the finite element analysis to compute the heat transfer between the fluid and structure. 


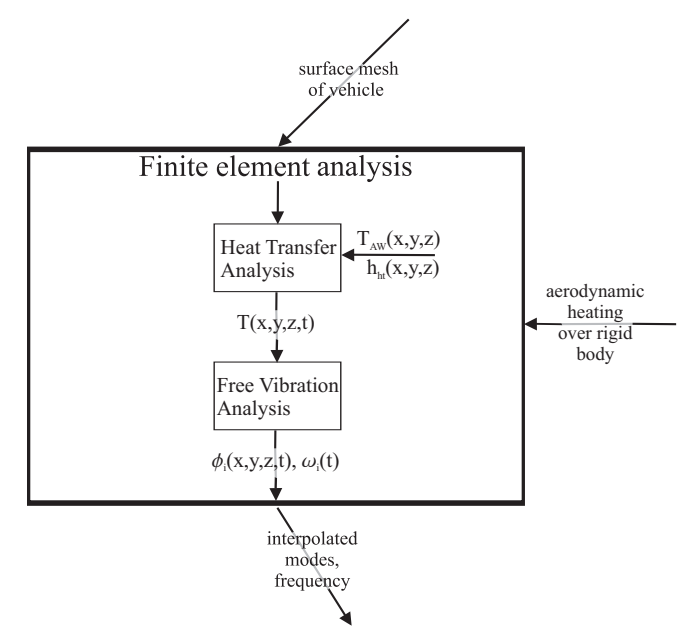

Figure 11. A flow diagram of the finite element analysis procedure when the heat transfer between the fluid and structure is included in the analysis.

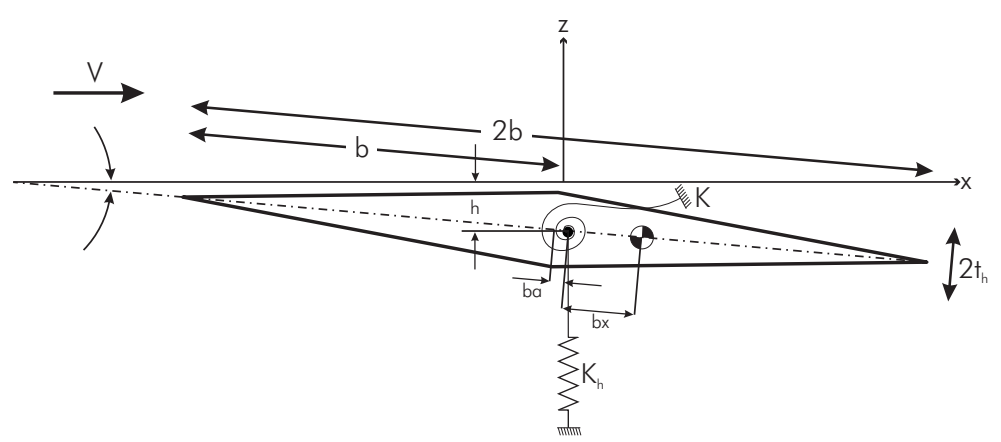

Figure 12. Two degree-of-freedom typical airfoil geometry. 


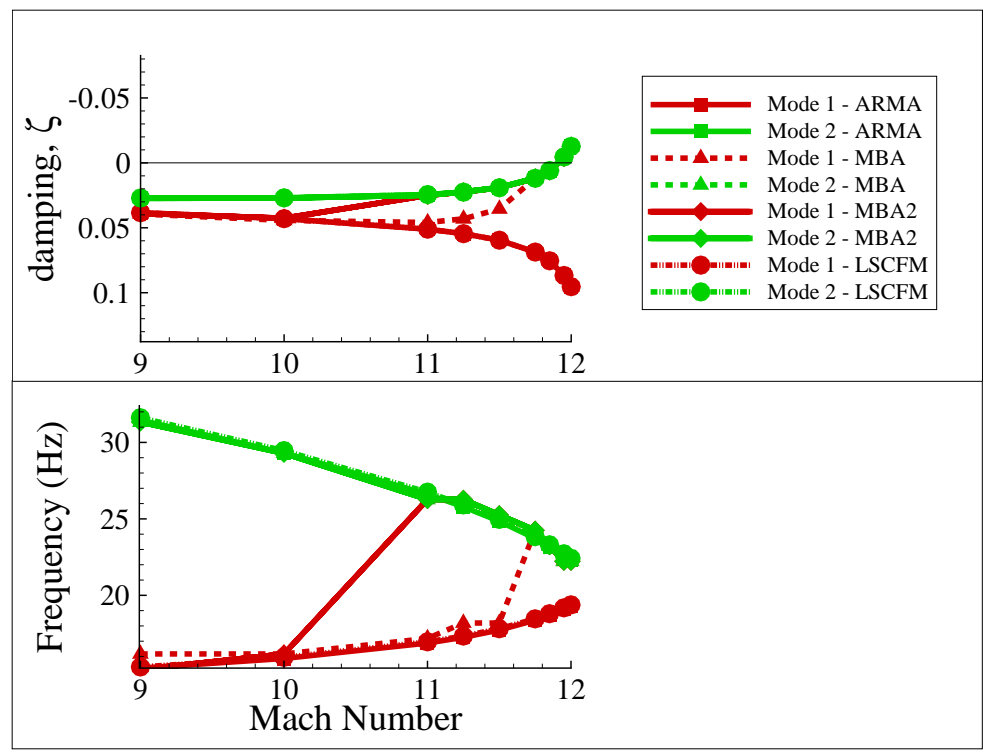

Figure 13. Aeroelastic behavior, as represented by damping and frequency, for a typical section using NavierStokes aerodynamics at $40,000 \mathrm{ft}$; predicted using different time domain identification techniques.
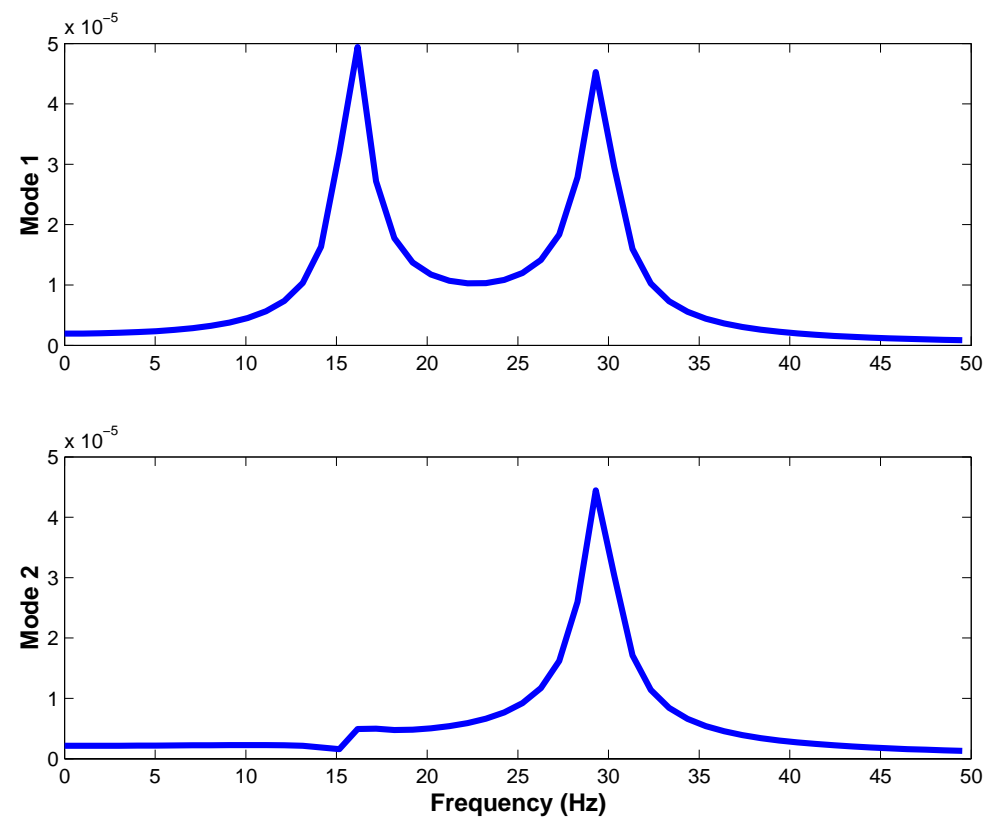

Figure 14. Frequency spectrum of the aeroelastic transients of a typical section at $M=10.0$ and $40,000 \mathrm{ft}$. 

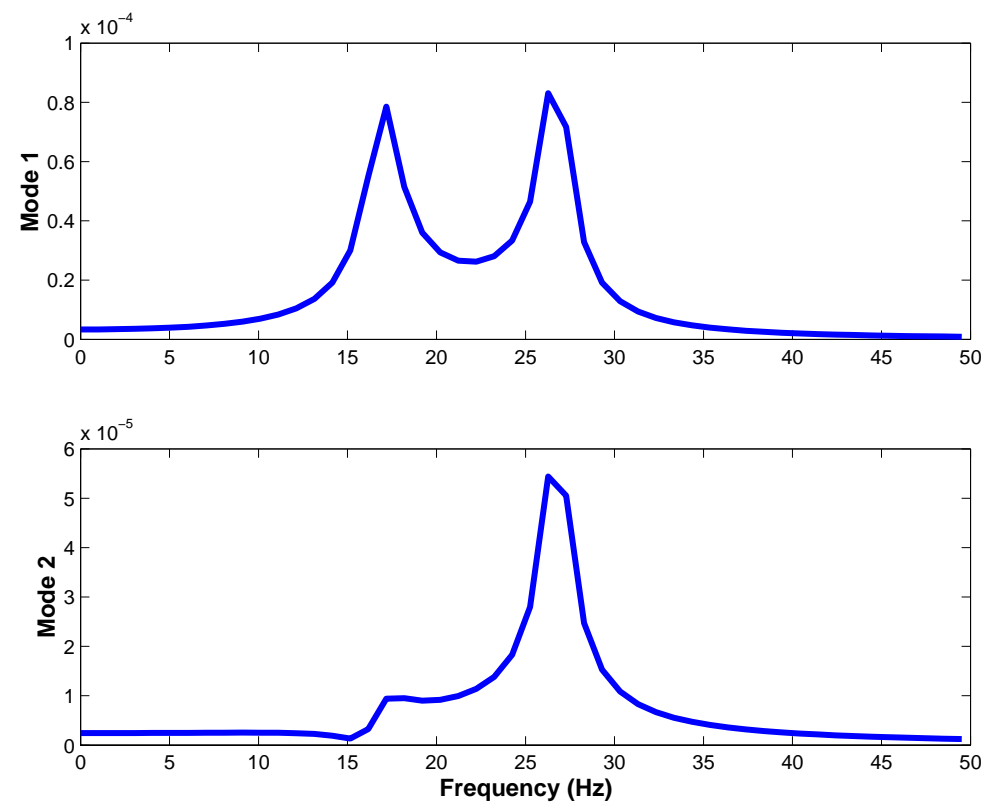

Figure 15. Frequency spectrum of the aeroelastic transients of a typical section at $M=11.0$ and $40,000 \mathrm{ft}$.

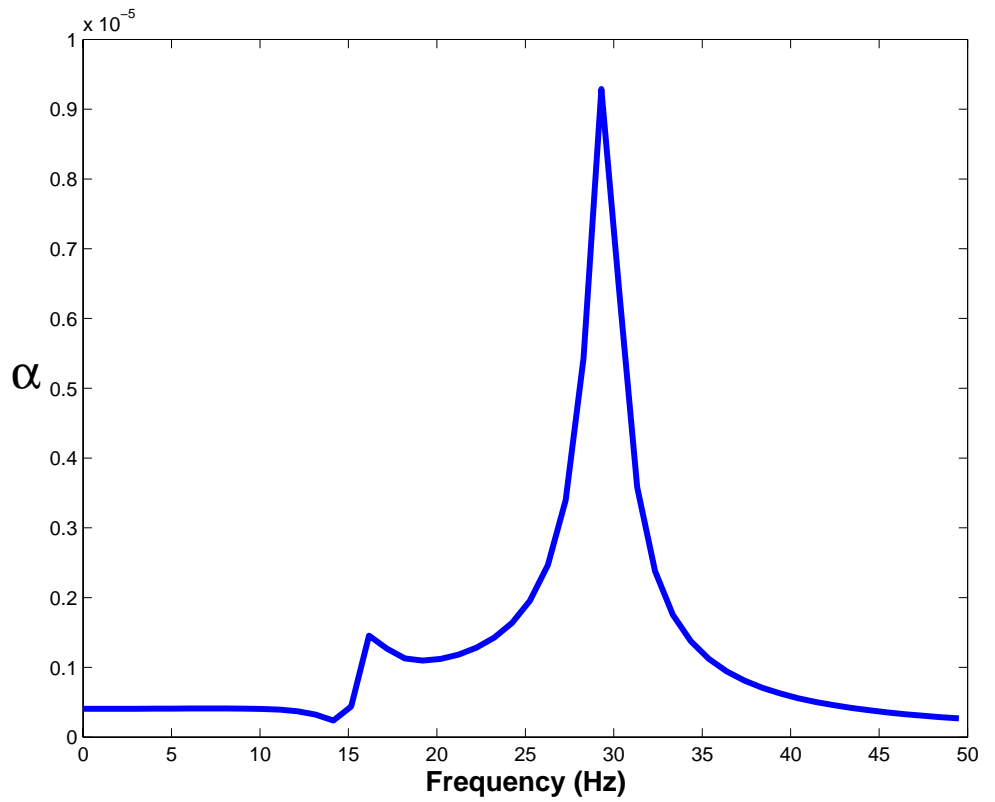

Figure 16. Frequency spectrum of the pitch response of a typical section at $M=11.0$ and $40,000 \mathrm{ft}$. 


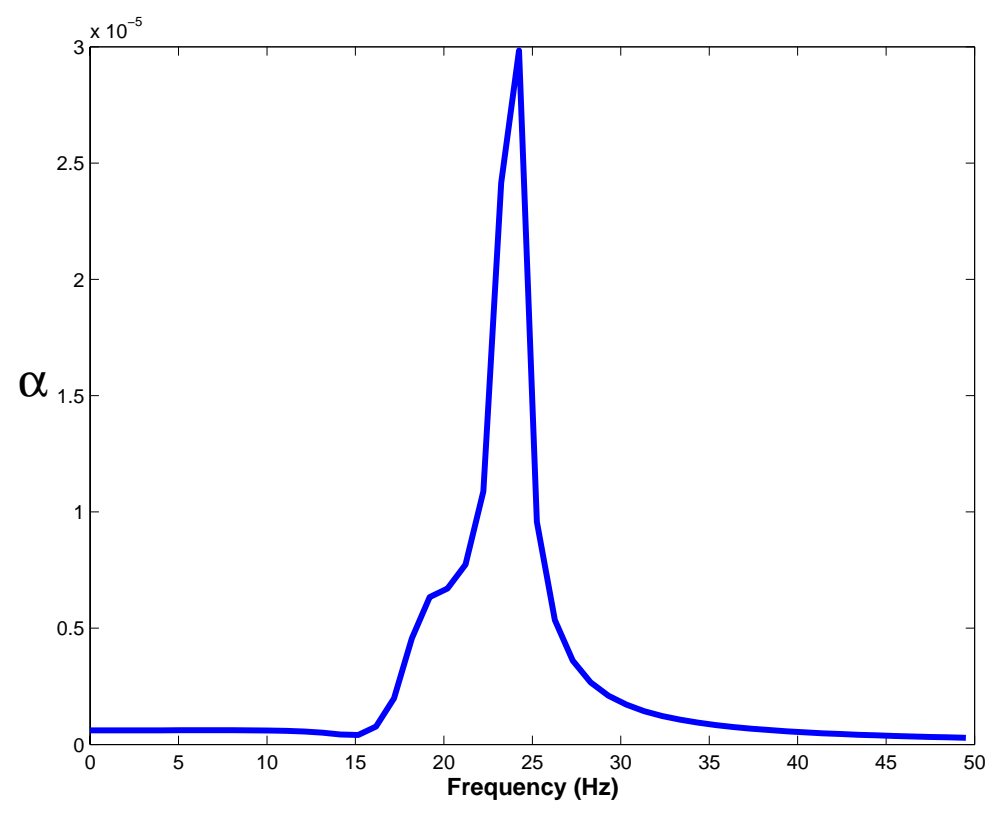

Figure 17. Frequency spectrum of the pitch response of a typical section at $M=11.75$ and 40,000ft.

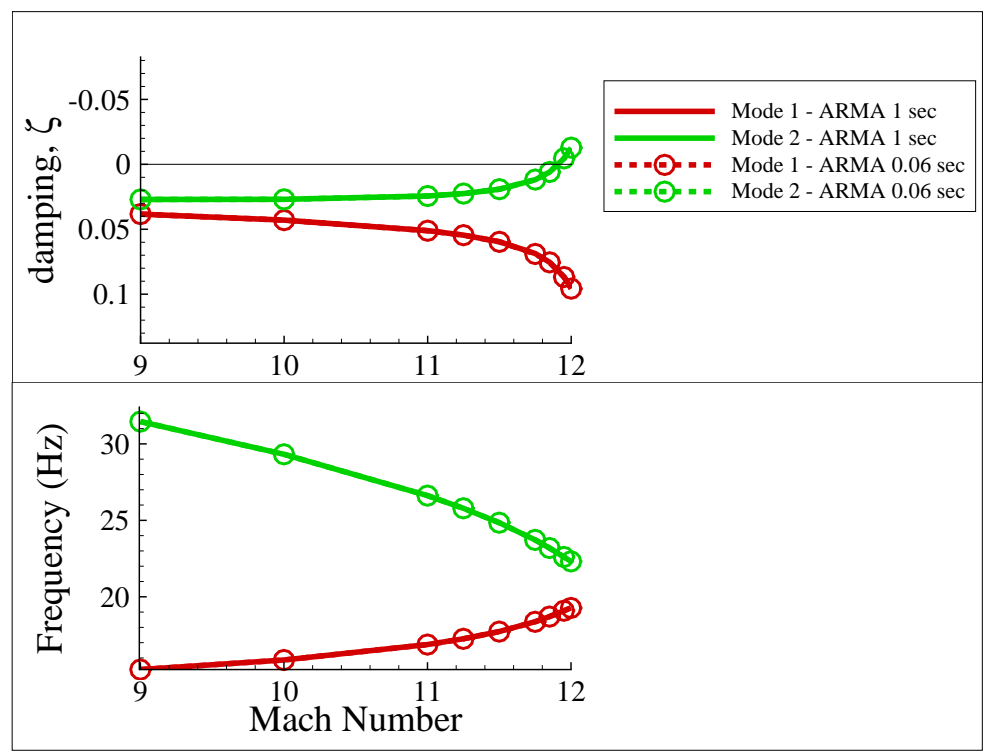

Figure 18. Aeroelastic behavior, of a typical section using Navier-Stokes aerodynamics, predicted using an ARMA model of the aeroelastic system and different time record lengths of the response, at 40,000 $\mathrm{ft}$. 


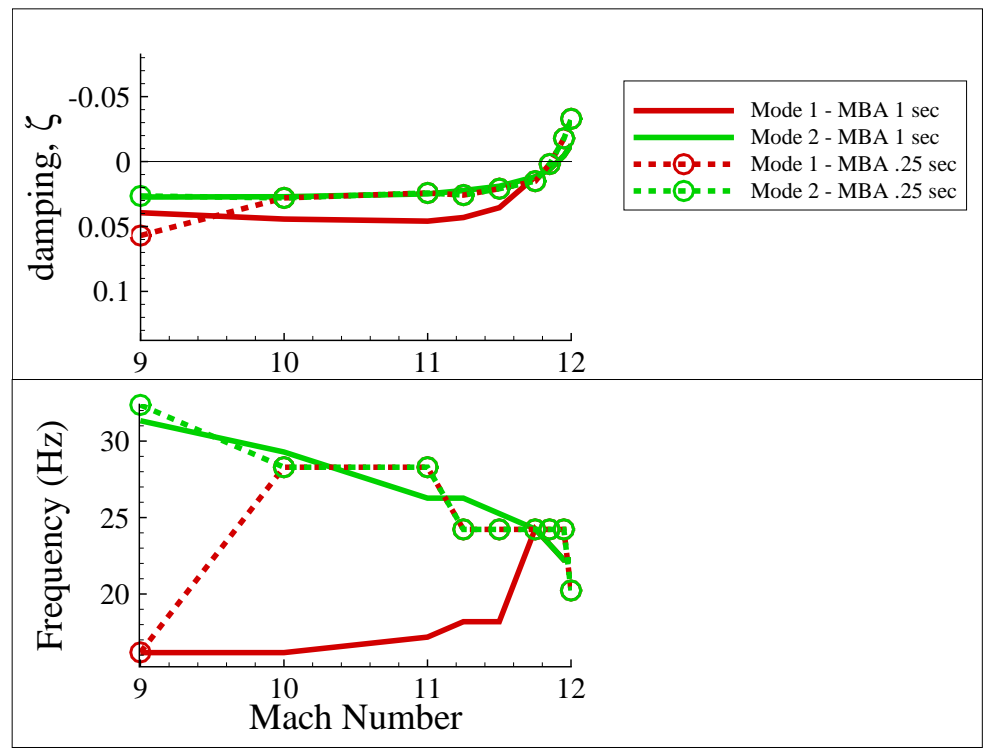

Figure 19. Aeroelastic behavior, of a typical section using Navier-Stokes aerodynamics, predicted using MBA and different time record lengths of the response, at 40,000 $\mathrm{ft}$.

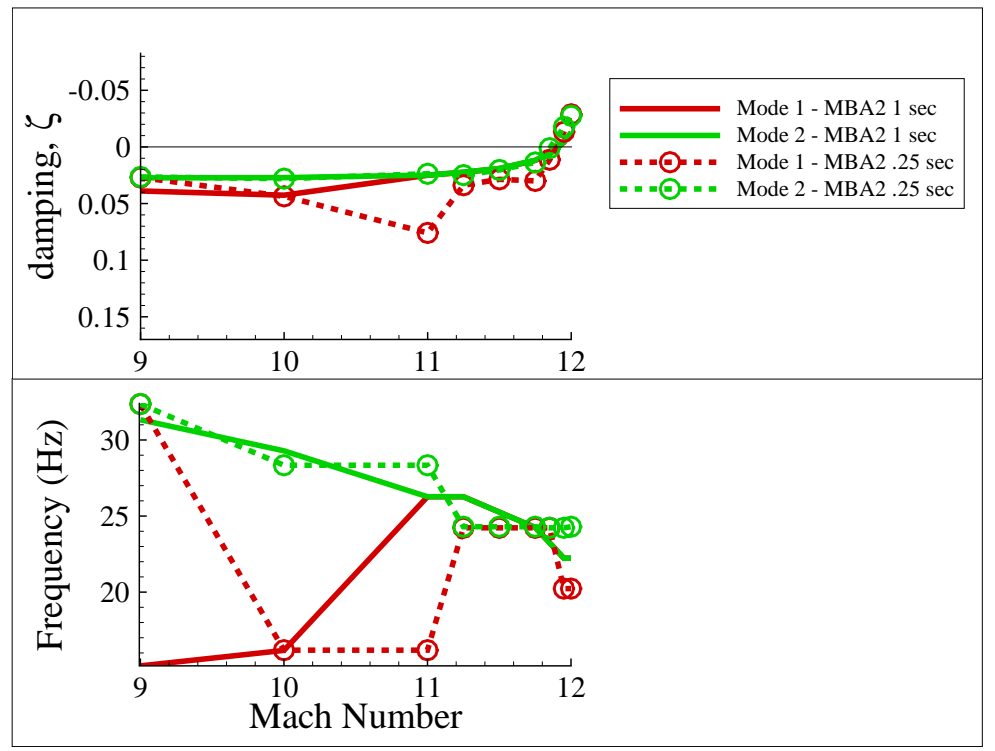

Figure 20. Aeroelastic behavior, of a typical section using Navier-Stokes aerodynamics, predicted using MBA2 and different time record lengths of the response, at $40,000 \mathrm{ft}$. 


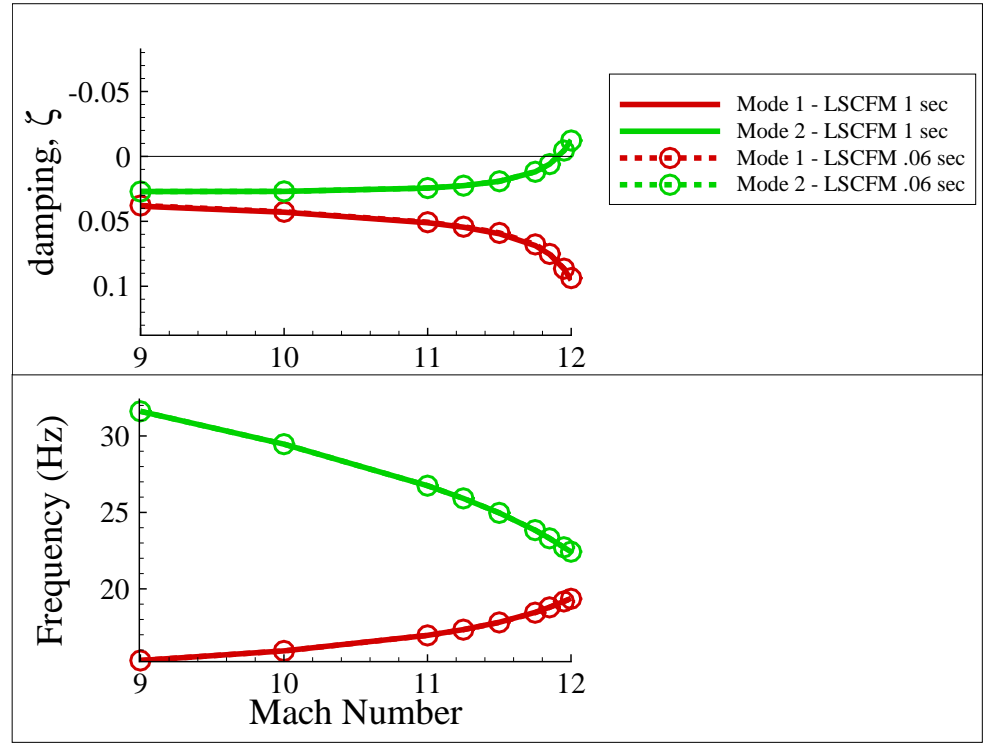

Figure 21. Aeroelastic behavior, of a typical section using Navier-Stokes aerodynamics, predicted using LSCFM and different time record lengths of the response, at $40,000 \mathrm{ft}$.

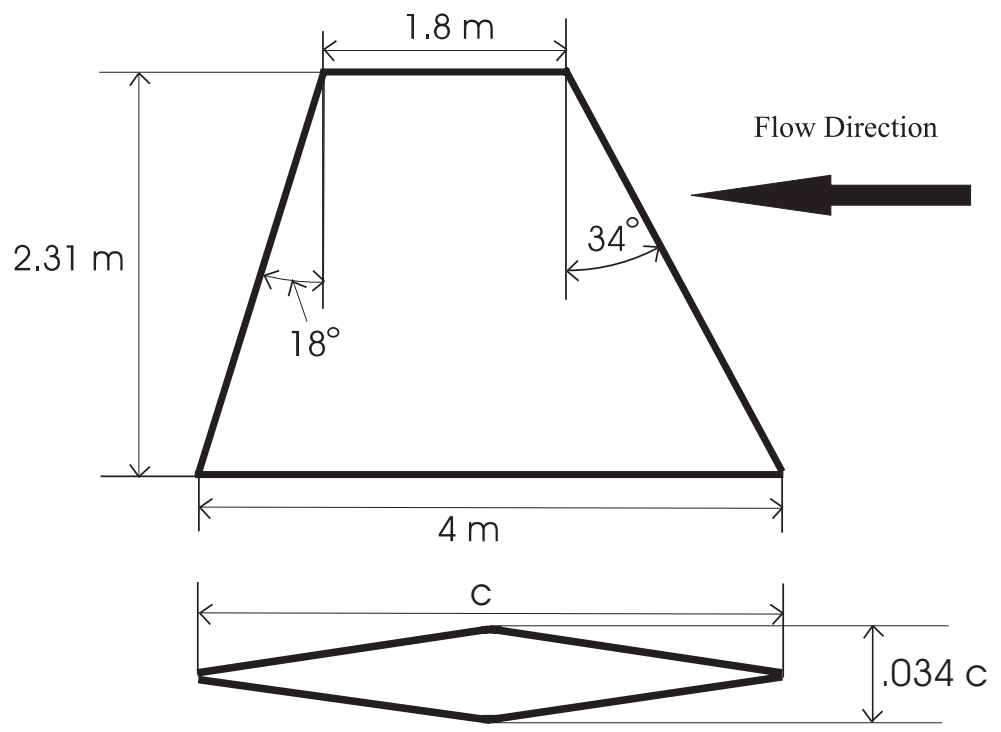

Figure 22. Planform and cross-sectional views of the low aspect ratio wing. 
Mode 1, 13.41 Hz.. First Bending.

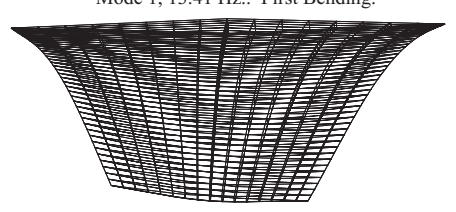

Mode 3, 49.18 Hz.. Second Bending.

Flow Direction
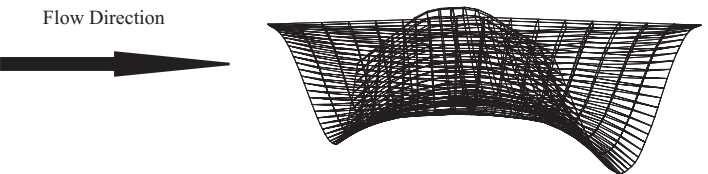

Mode 4, 77.14 Hz.. Second Torsional.

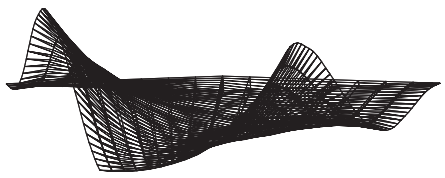

Mode2, $37.51 \mathrm{~Hz}$.. First Torsional.

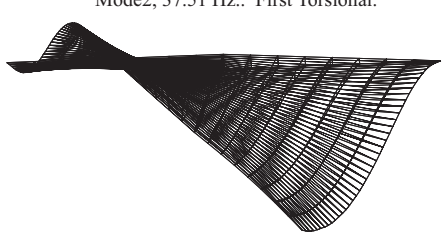




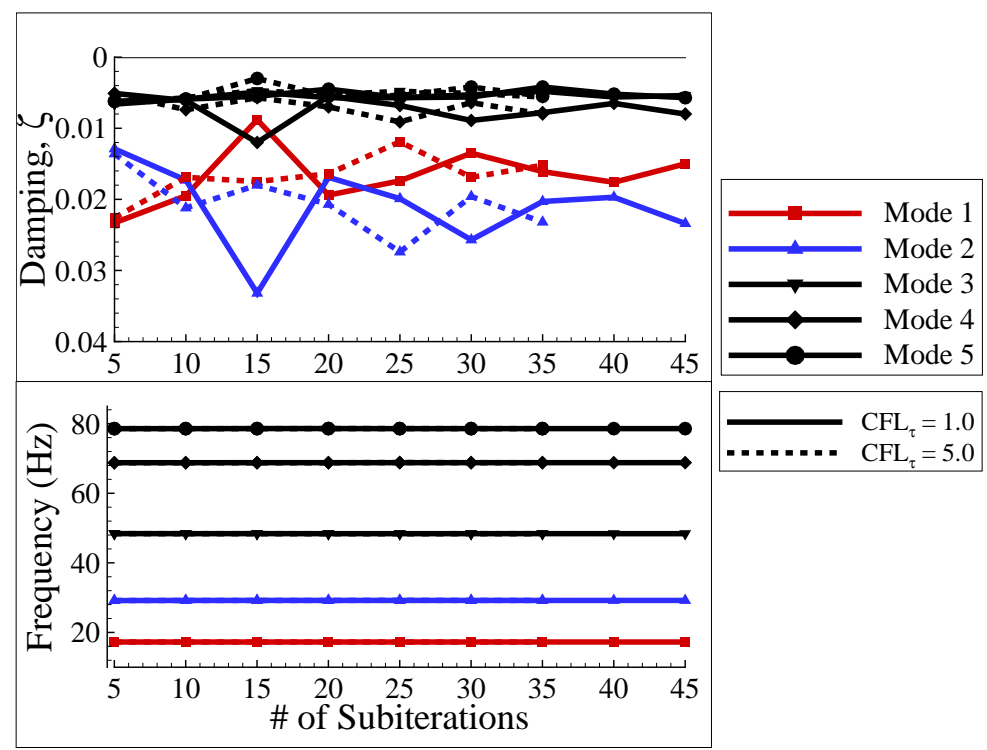

Figure 25. Effect of increasing sub-iterations and $C F L_{\tau}$ on the aeroelastic behavior of the low aspect ratio wing using Euler aerodynamics at $40,000 \mathrm{ft}$. Symbols indicate the mode number, while the solid vs. dashed lines indicate the value of $C F L_{\tau}$.

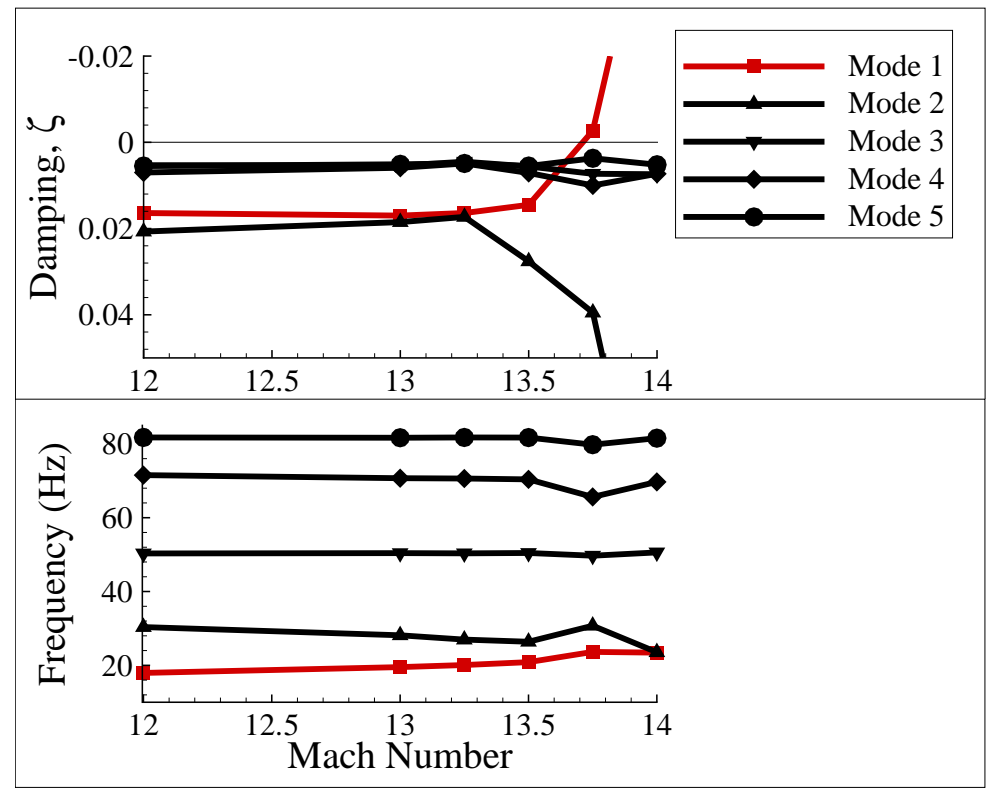

Figure 26. Aeroelastic behavior of the low aspect ratio wing using Euler aerodynamics at 40,000 ft. The results were generated using a $0.63 \times 10^{6}$ cell grid. 


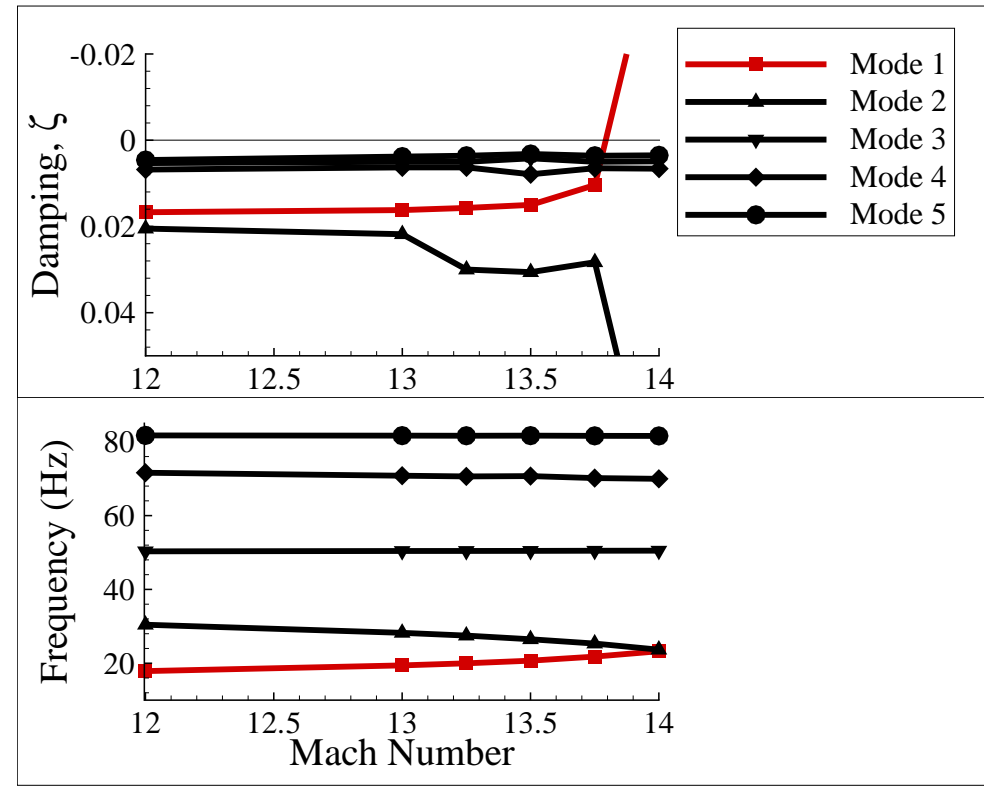

Figure 27. Aeroelastic behavior of the low aspect ratio wing using Euler aerodynamics at 40,000 $\mathrm{ft}$. The results were generated using a $0.27 \times 10^{6}$ cell grid.
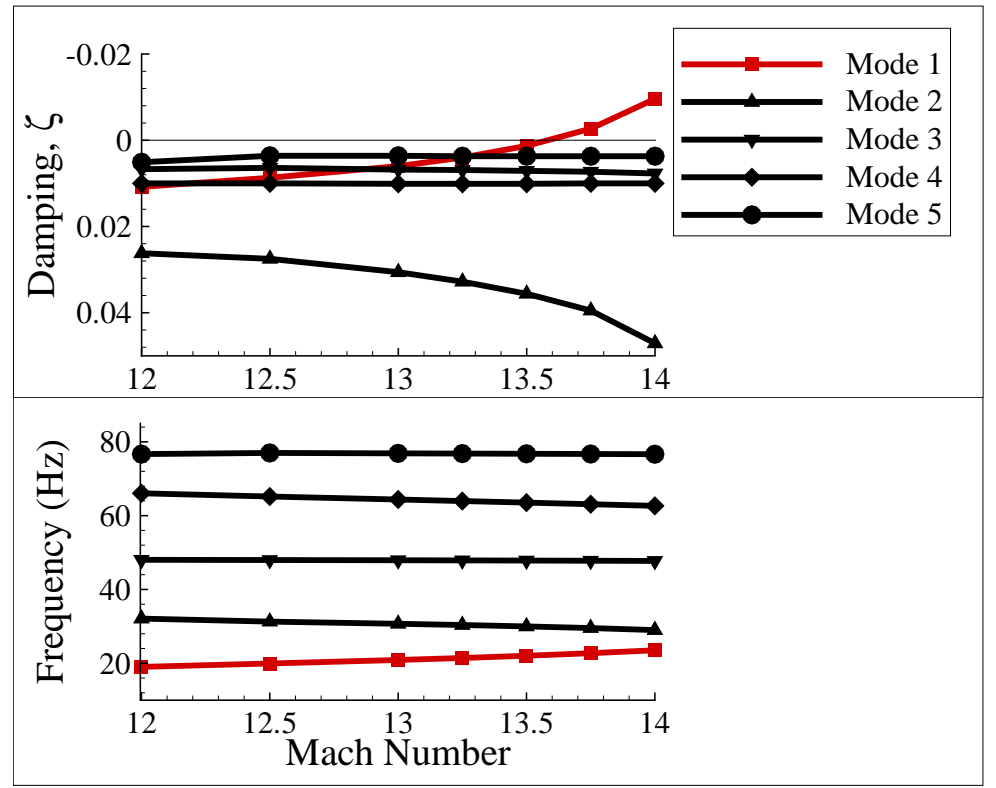

Figure 28. Aeroelastic behavior of the low aspect ratio wing using Navier-Stokes aerodynamics at $40,000 \mathrm{ft}$, $C F L_{\tau}=\mathbf{1 . 0}$ 

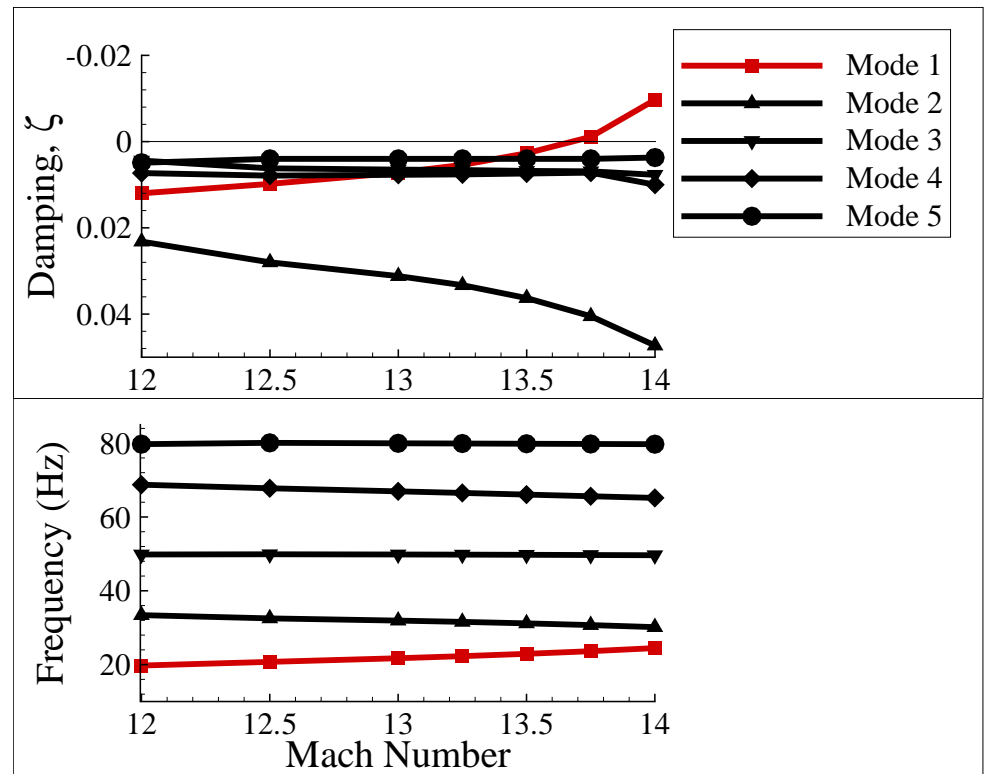

Figure 29. Aeroelastic behavior of the low aspect ratio wing using Navier-Stokes aerodynamics at $40,000 \mathrm{ft}$, $C F L_{\tau}=\mathbf{5 . 0}$

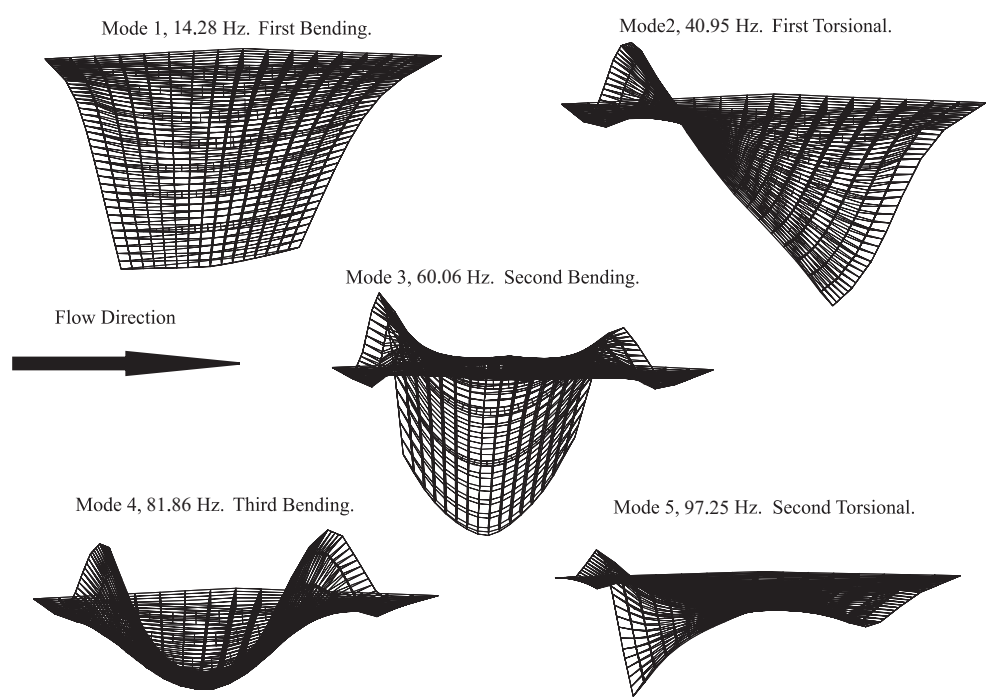

Figure 30. First 5 free vibration modes of the modified low aspect ratio wing 

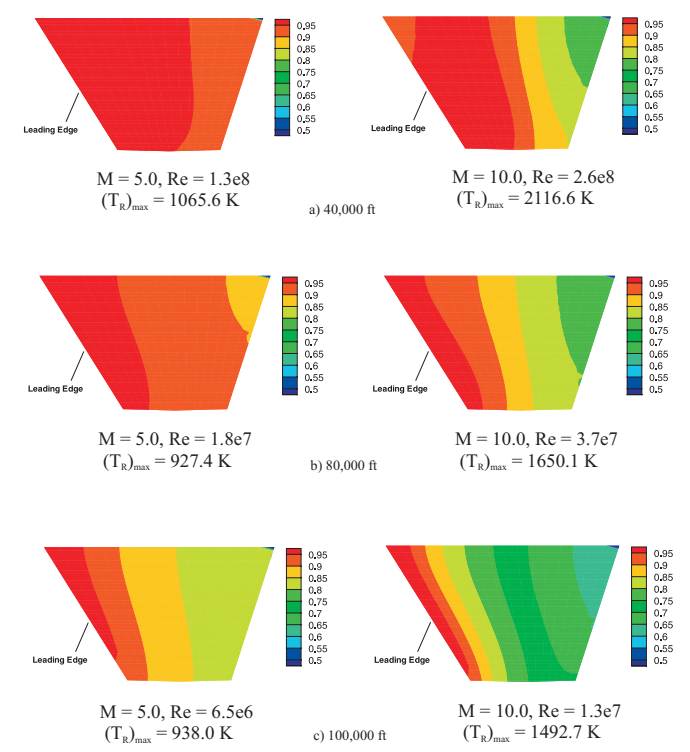

Figure 31. Normalized radiation equilibrium wall temperatures of the modified low aspect ratio wing for various altitudes and free stream Mach numbers.

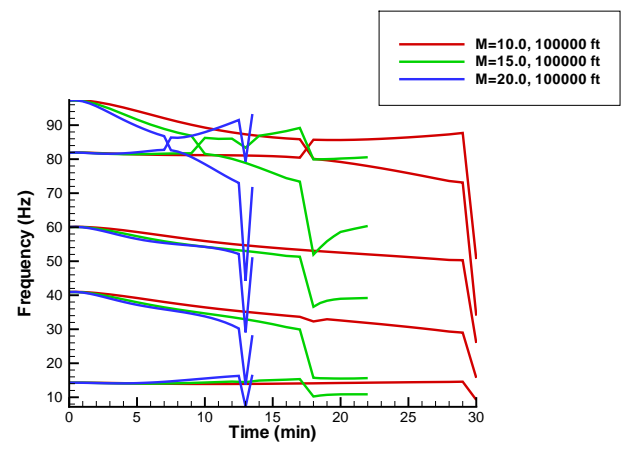

Figure 32. Natural frequencies of the modified low-aspect ratio wing subject to aerodynamic heating at 100,000 ft. 


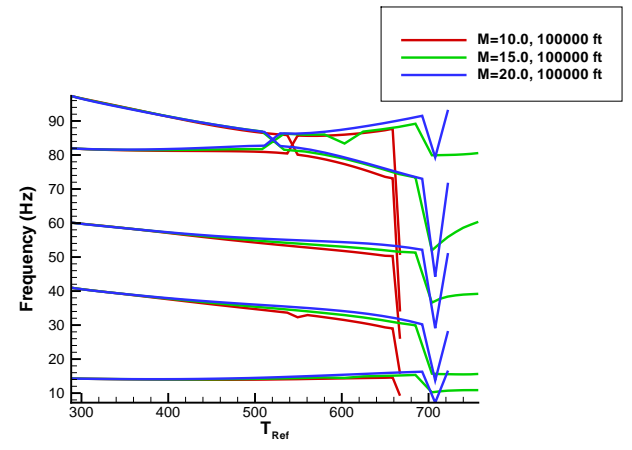

Figure 33. Natural frequencies of the modified low-aspect ratio wing subject to aerodynamic heating at 100,000 ft. Frequencies are plotted as a function of $T_{R e f}$, which corresponds to the leading edge temperature of the $75 \%$ span location.

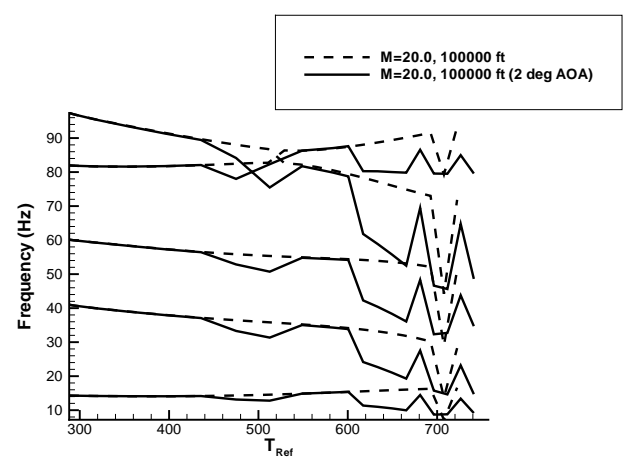

Figure 34. Natural frequencies of the modified low-aspect ratio wing subject to aerodynamic heating, and varying angle of attack at $100,000 \mathrm{ft}$. Frequencies are plotted as a function of $T_{R e f}$, which corresponds to the leading edge temperature of the $75 \%$ span location. 


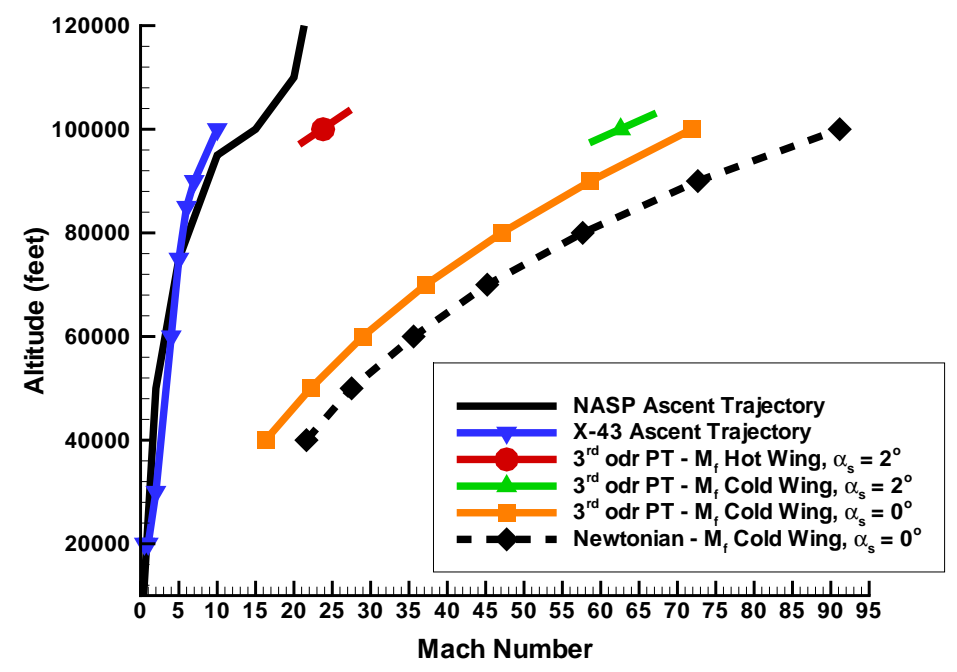

Figure 35. Comparison of flutter boundaries for the modified low aspect ratio wing for the ascent trajectories of typical hypersonic aircraft. The flutter boundary for the heated wing at $\alpha_{s}=2^{\circ}$ corresponds to $T_{R e f}=665$ $\mathrm{K}$ in Figure 34.

Mode 1: $5.27 \mathrm{~Hz}$. Symmetric Fin Bending

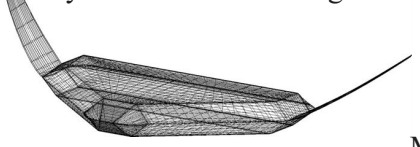

Mode 3: $11.12 \mathrm{~Hz}$

First Fuselage Bending

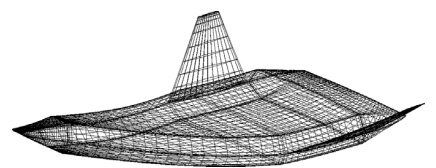

Mode 4: $14.59 \mathrm{~Hz}$.

First Fuselage Torsion

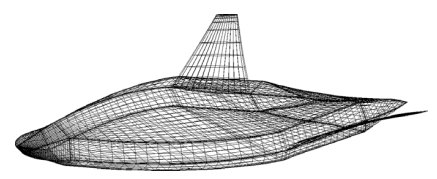

Mode 2: $5.38 \mathrm{~Hz}$.

Asymmetric Fin Bending

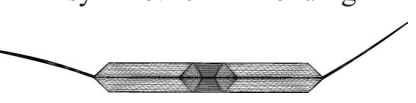

Mode 5: $21.95 \mathrm{~Hz}$. Second Fuselage Bending

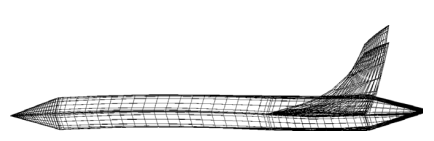

Figure 36. First 5 free vibration modes of the generic reusable launch vehicle.. 


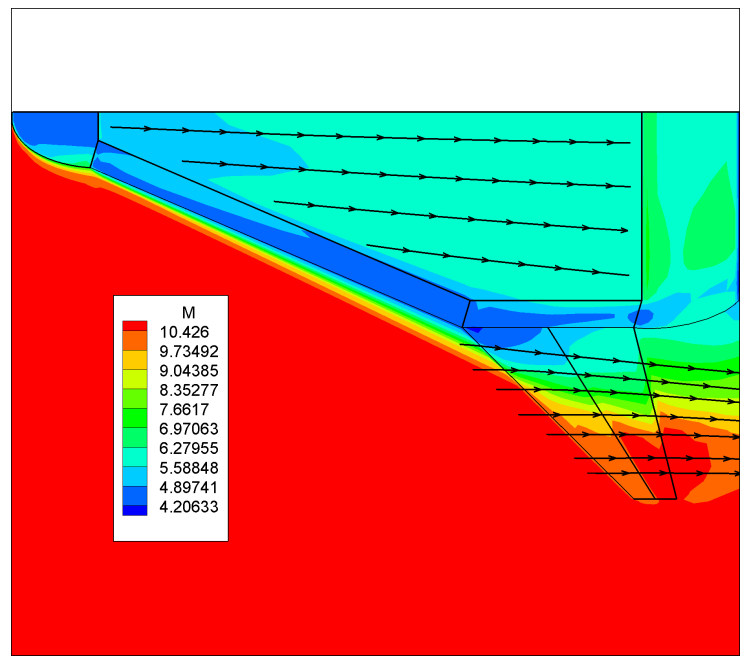

Figure 37. Streamlines and Mach contours on the generic vehicle using Euler aerodynamics, top view, $M=10.5$, 40,000 feet.

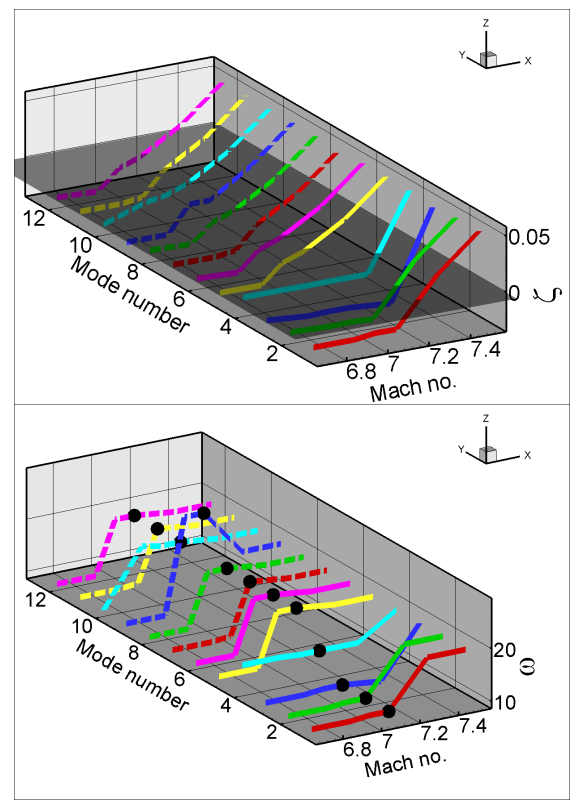

Figure 38. Aeroelastic behavior of the generic vehicle at 40,000 feet, calculated using nonlinear piston theory, and 12 modes. 


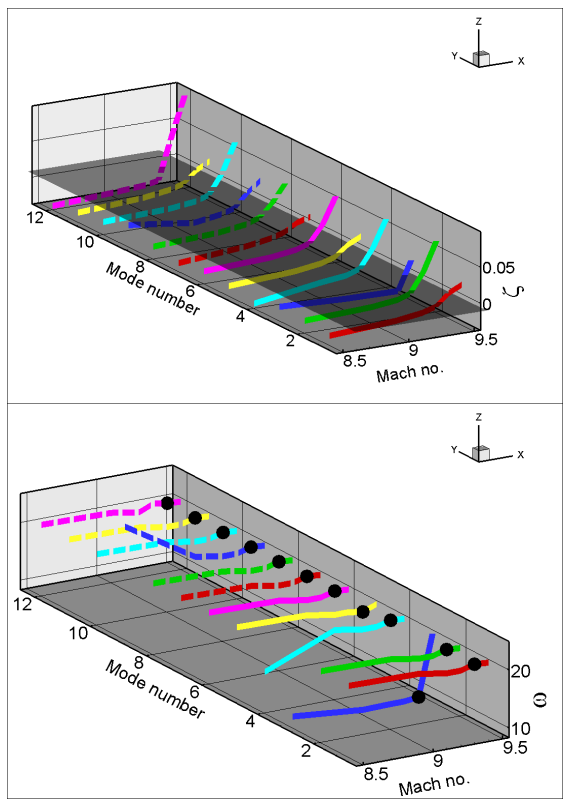

Figure 39. Aeroelastic behavior of the generic vehicle at 40,000 feet, calculated using Euler aerodynamics, and 12 modes. The calculations were carried out on the coarse mesh.

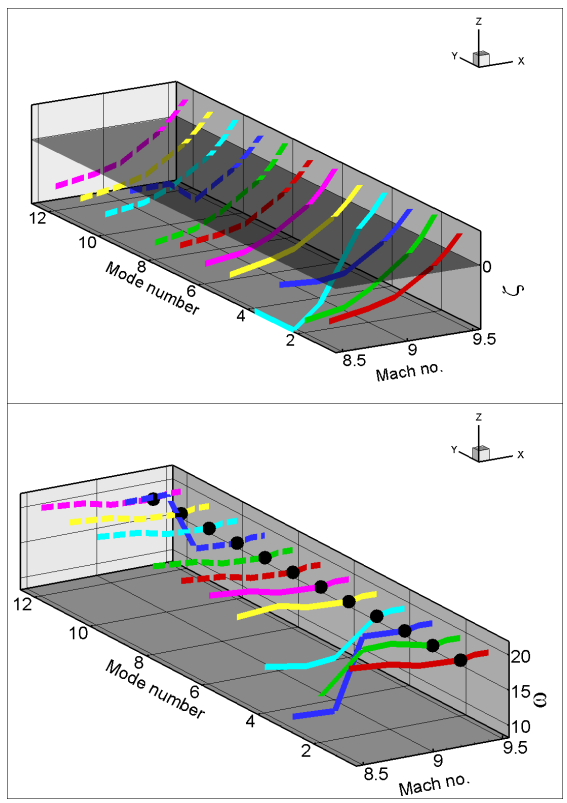

Figure 40. Aeroelastic behavior of the generic vehicle at 40,000 feet, calculated using Euler aerodynamics, and 12 modes. The calculations were carried out on the fine mesh. 


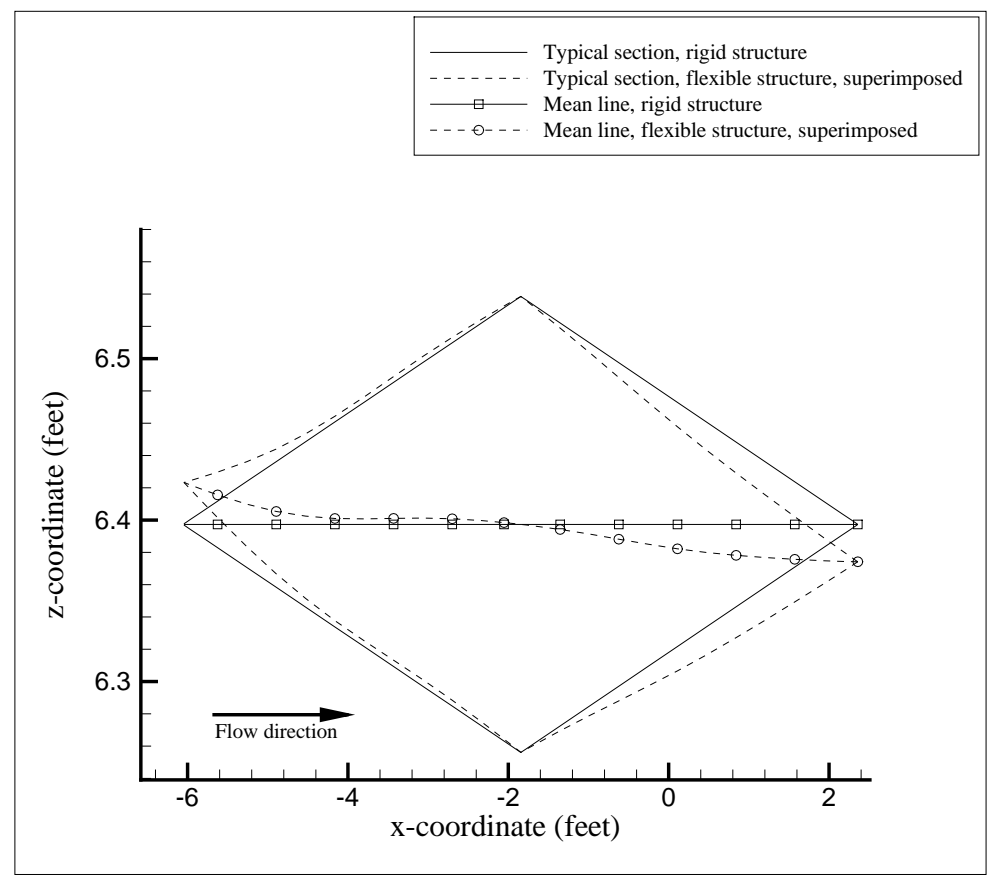

Figure 41. Superimposed views of the typical sections and mean lines for the rigid and damped equilibrium configurations of the typical cross-section. $M=8.0,40,000$ feet.

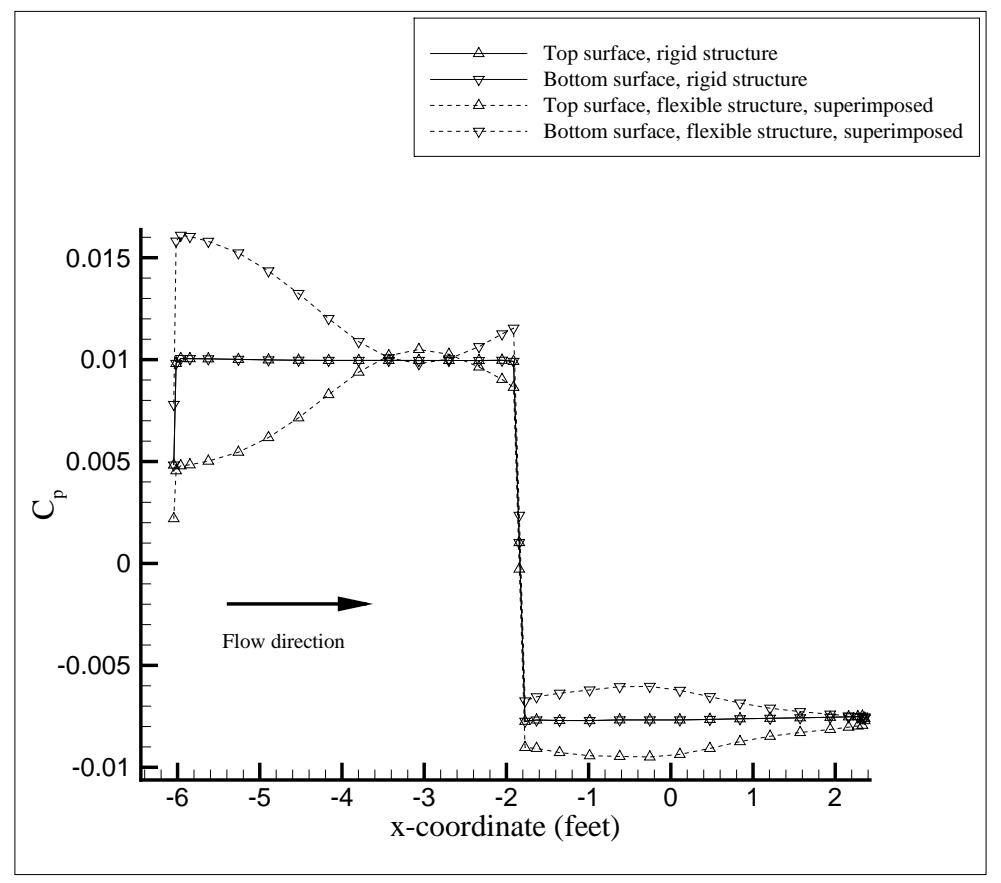

Figure 42. $C_{p}$ on the surface for the rigid and damped equilibrium configurations of the typical cross-section. $M=8.0,40,000$ feet. 


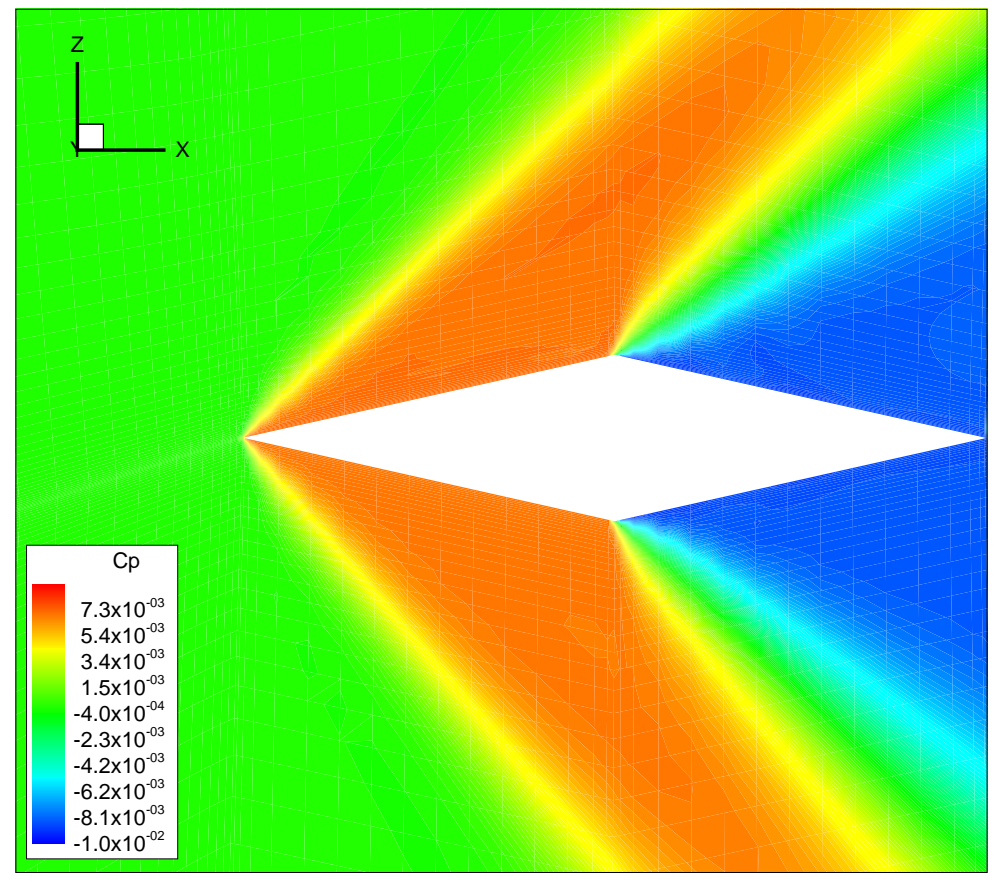

Figure 43. Pressure contours around the rigid typical section of the canted fin of the generic vehicle at $M=8.0$, at 40,000 feet, using Euler aerodynamics.

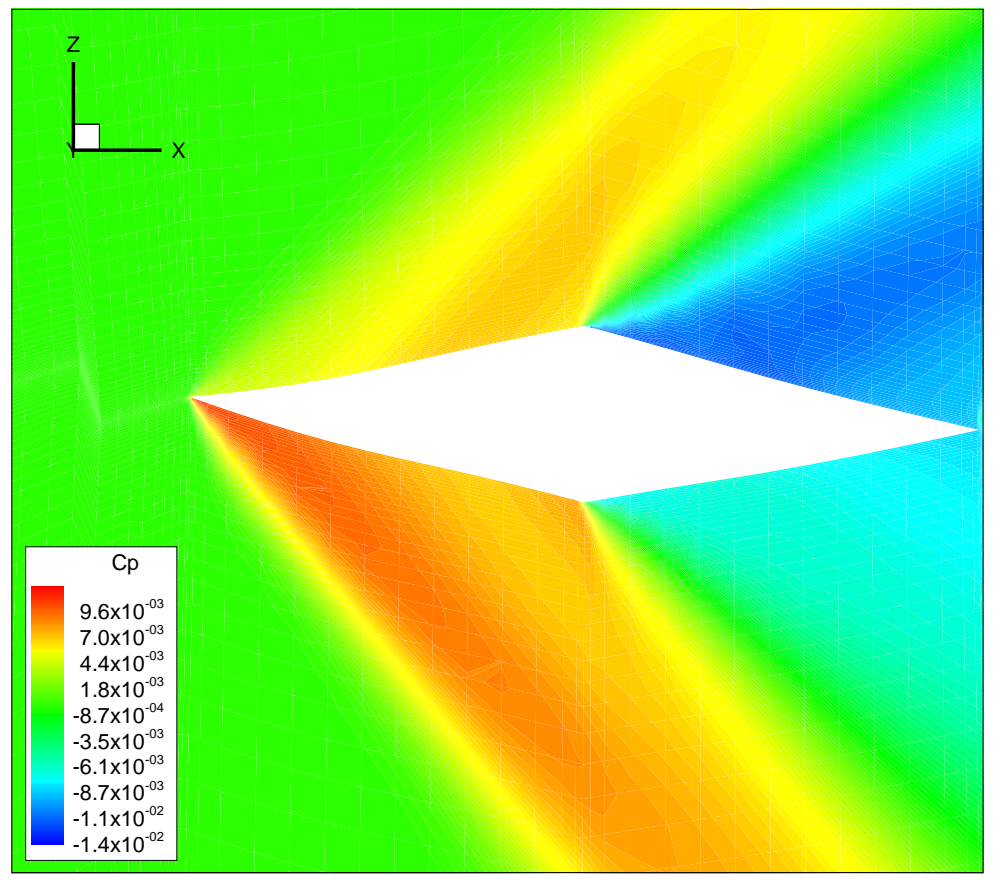

Figure 44. Pressure contours around the deformed typical section of the canted fin of the generic vehicle at $M=8.0$, at 40,000 feet, using Euler aerodynamics. 


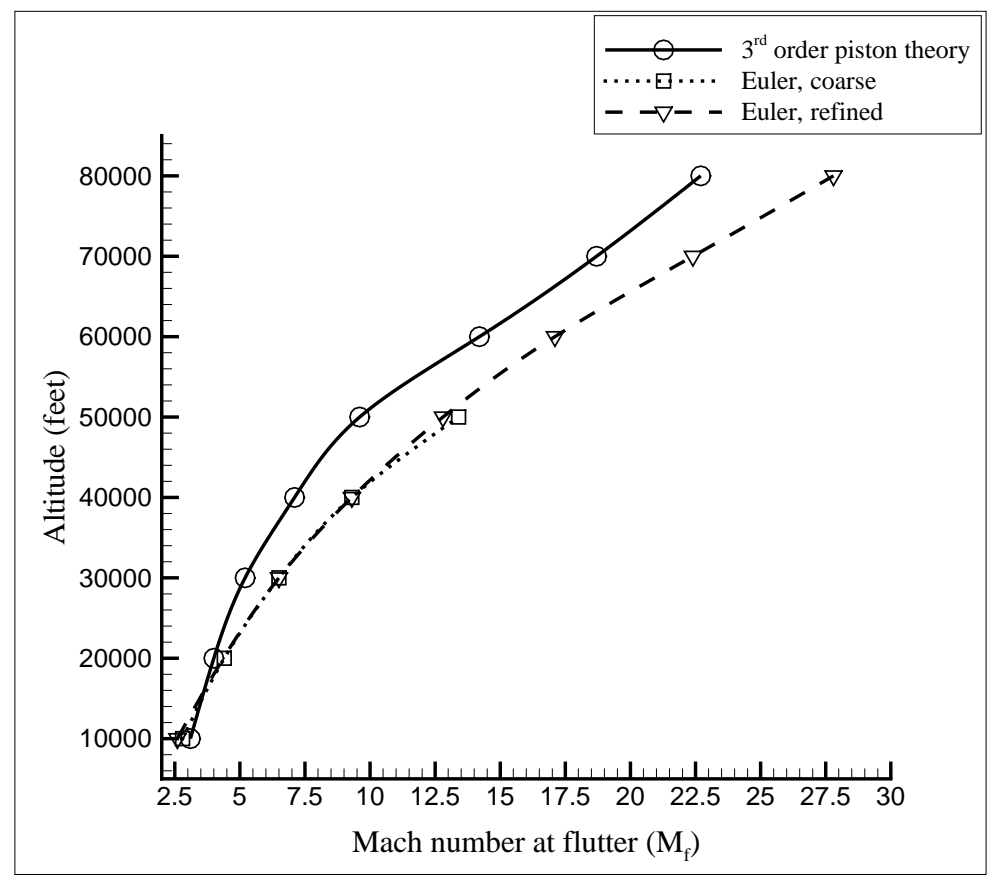

Figure 45. Flutter envelope of the generic hypersonic vehicle, calculated using piston theory and Euler aerodynamics.

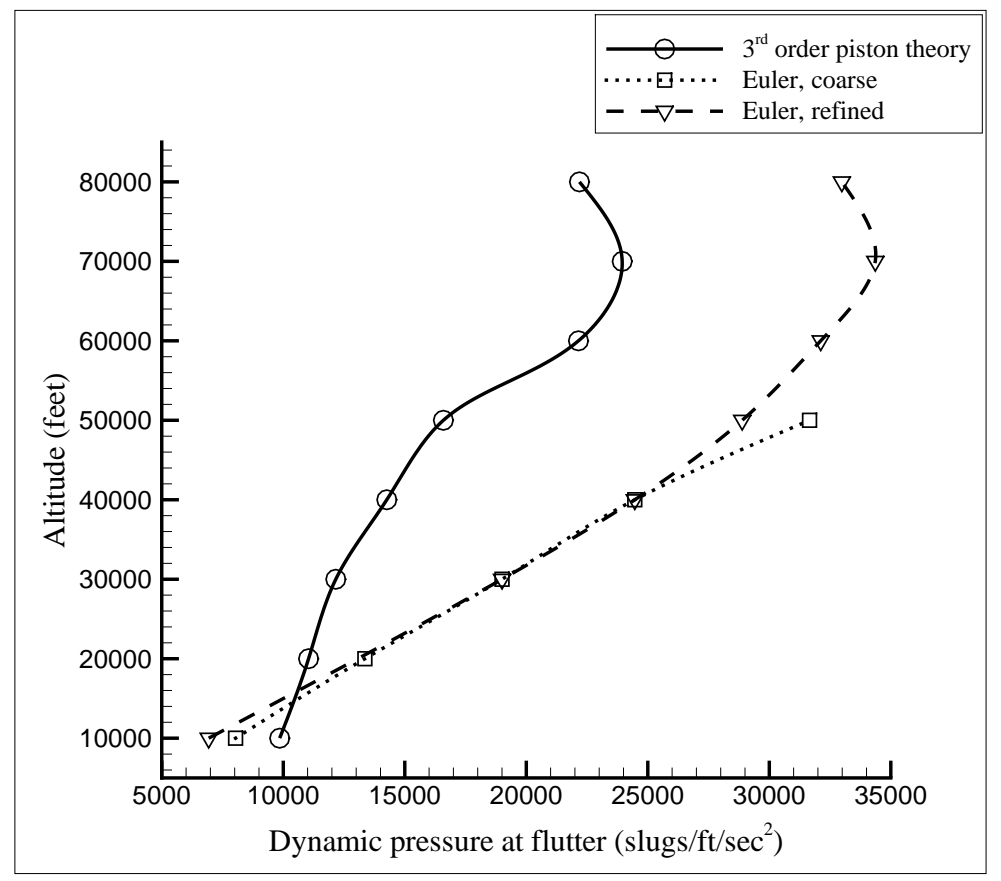

Figure 46. Dynamic pressure at flutter for the generic vehicle, calculated using piston theory and Euler aerodynamics. 


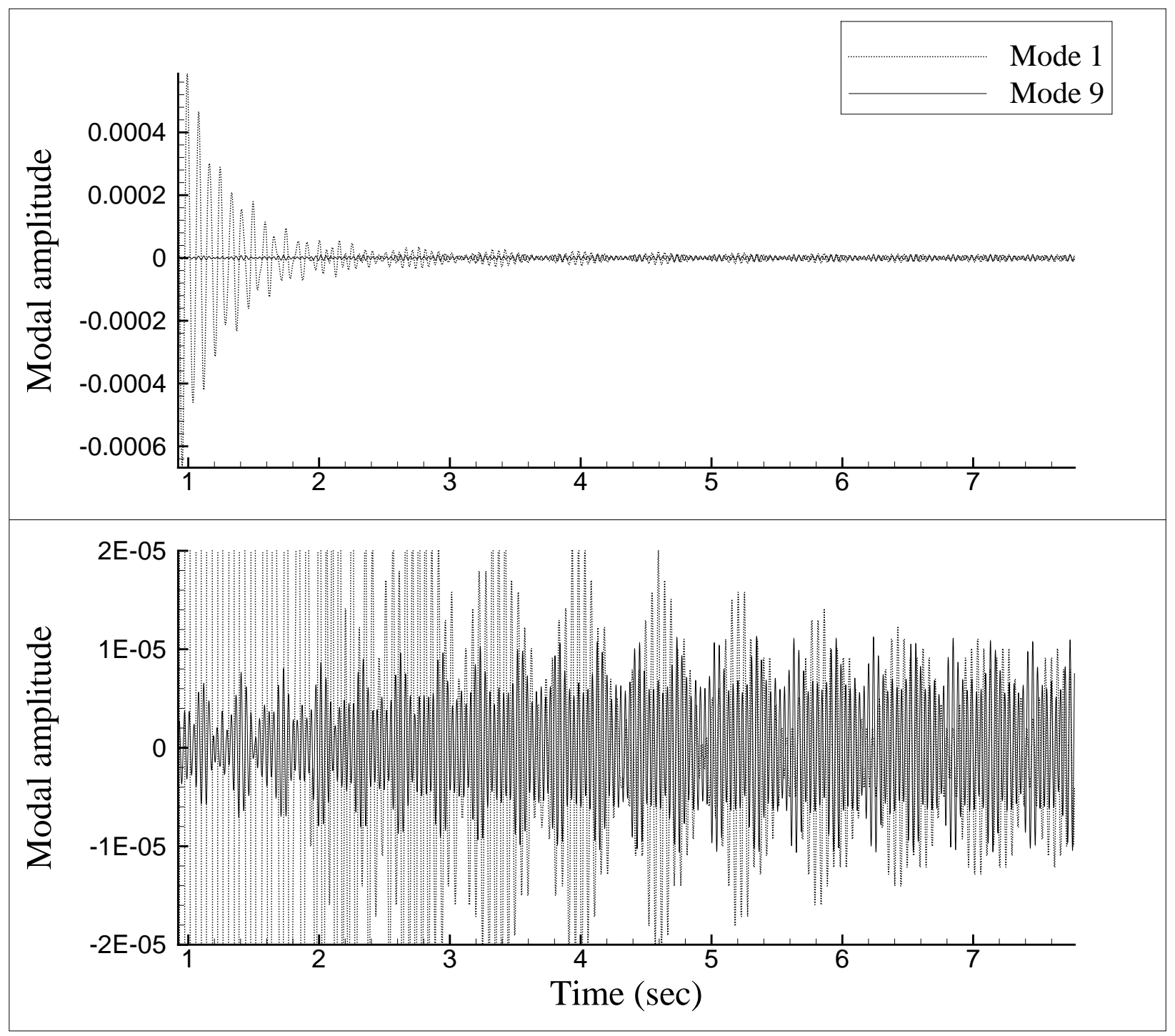

Figure 47. Aeroelastic transients for the generic vehicle at $M=\mathbf{2 8 . 0}$ and 80,000 feet. Only modes 1 and 9 are shown for clarity. 